\title{
Supporting Information for \\ Synthesis of High Oxidation State Bimetallic Alkylidene Complexes for Controlled ROMP Synthesis of Triblock Copolymers
}

Richard R. Schrock*, Andrea J. Gabert, Rojendra Singh, and Adam S. Hock

For 1b, 3, and 4 .

1. Fully labeled ORTEP drawing

2. Crystal data and structure refinement

3. Atomic coordinates

4. Bond lengths and angles

5. Anisotropic thermal parameters 


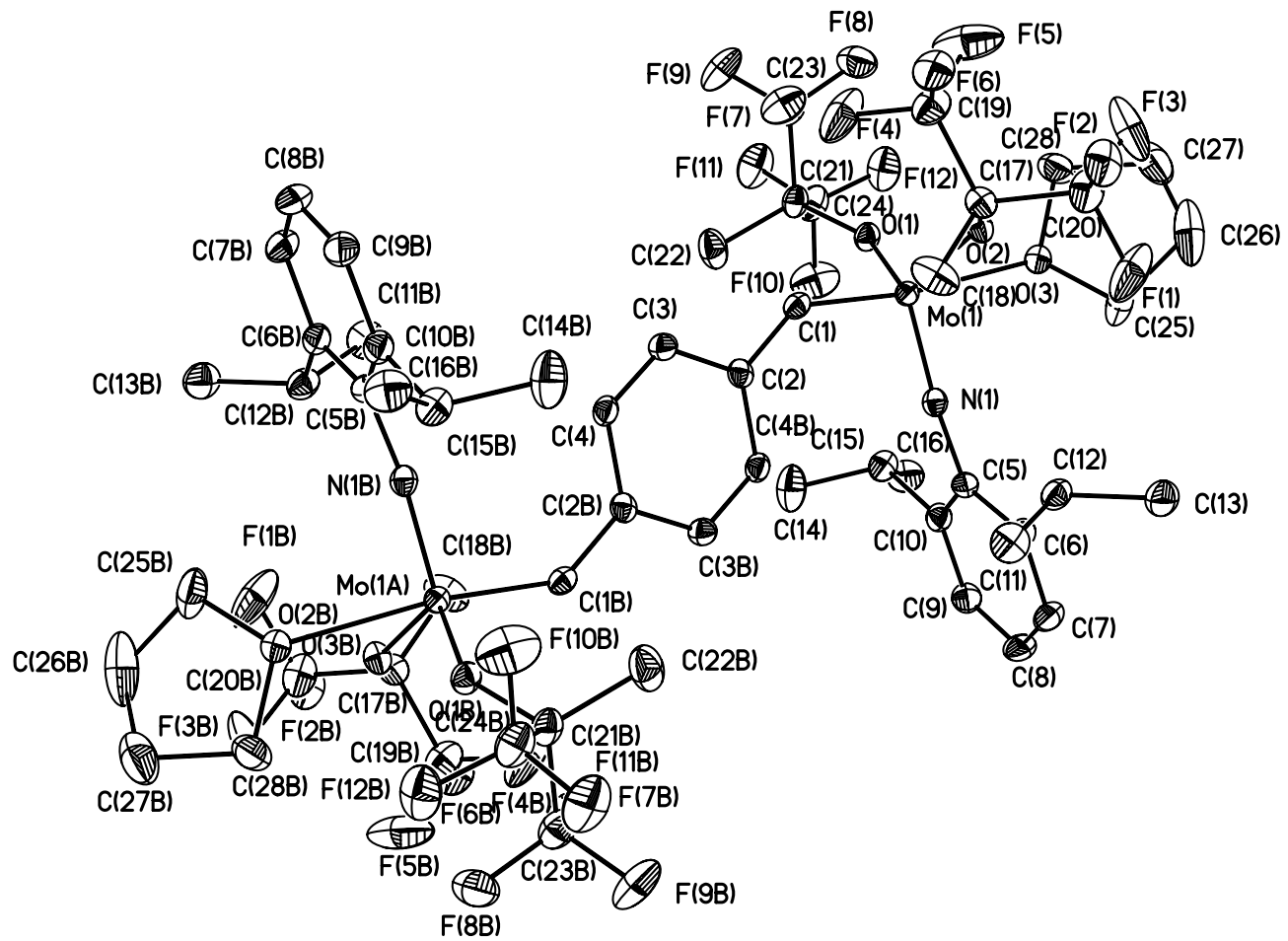

Figure 1. Thermal Ellipsoid Plot (50\% probability level) of the structure of $\mathbf{1 b}$. Hydrogens have been omitted for clarity. A second, chemically equivalent, but crystallographically indenpedent molecule is also present. It is labeled similarly with the trailing letter "A". 


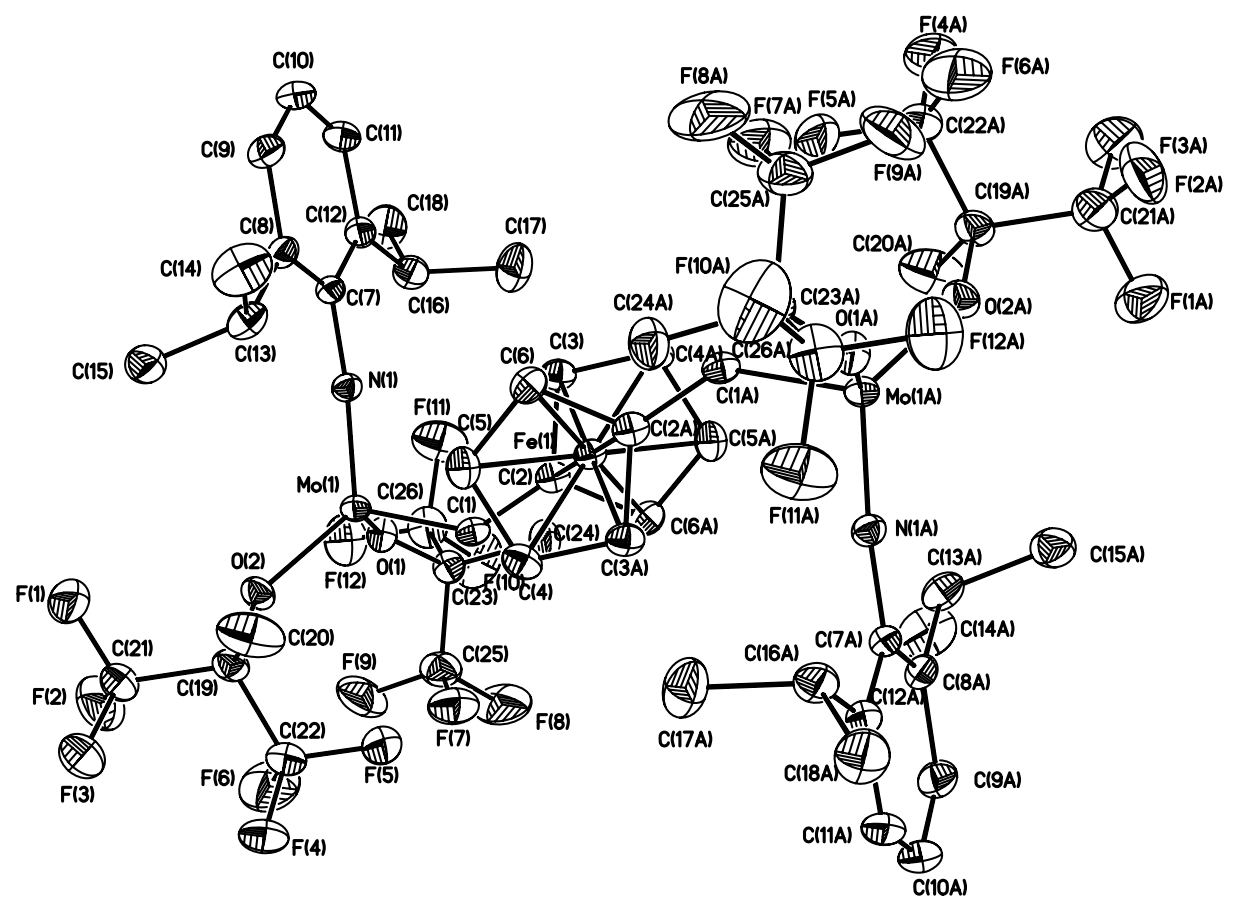

Figure 2. Thermal Ellipsoid Plot (50\% probability level) of the structure of 3. Hydrogens have been omitted for clarity. 


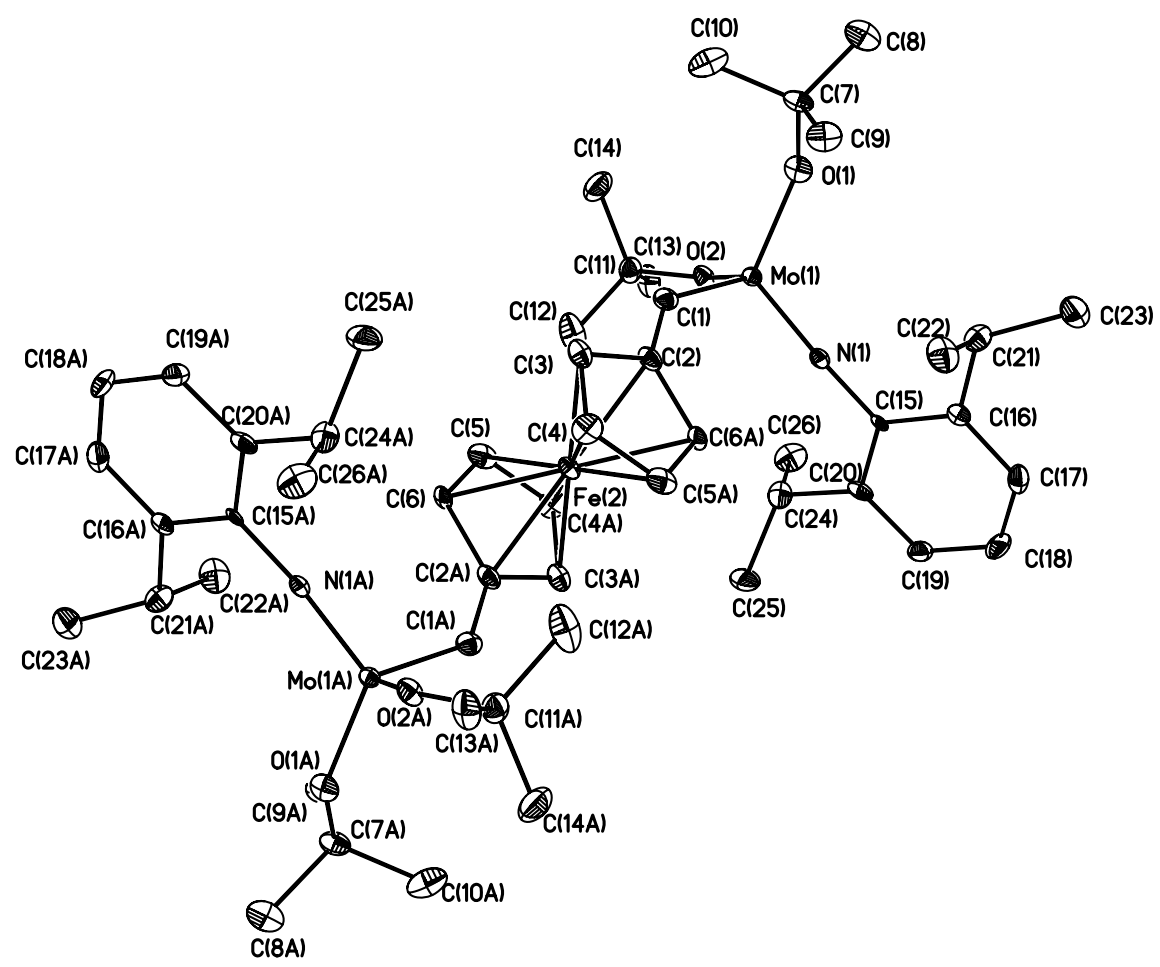

Figure 3 Thermal Ellipsoid Plot (50\% probability level) of the structure of 4 . Hydrogens have been omitted for clarity. 
Table 1. Crystal data and structure refinement for $\mathbf{1 b .}$

Empirical formula

Formula weight

Temperature

Wavelength

Crystal system

Space group

Unit cell dimensions

Volume

Z

Density (calculated)

Absorption coefficient

$\mathrm{F}(000)$

Crystal size

Theta range for data collection

Index ranges

Reflections collected

Independent reflections

Completeness to theta $=26.37^{\circ}$

Absorption correction

Max. and min. transmission

Refinement method

Data / restraints / parameters

Goodness-of-fit on $\mathrm{F}^{2}$

Final R indices [I $>2$ sigma(I)]

$\mathrm{R}$ indices (all data)

Largest diff. peak and hole
C56 H68 F24 Mo2 N2 O6

1513.00

100(2) K

$0.71073 \AA$

Triclinic

P1

$$
\begin{array}{ll}
\mathrm{a}=13.0696(19) \AA & \alpha=110.582(3)^{\circ} . \\
\mathrm{b}=15.465(2) \AA & \beta=93.113(2)^{\circ} . \\
\mathrm{c}=16.682(3) \AA & \gamma=91.248(2)^{\circ} .
\end{array}
$$

3149.0(8) $\AA^{3}$

2

$1.596 \mathrm{Mg} / \mathrm{m}^{3}$

$0.518 \mathrm{~mm}^{-1}$

1532

$0.28 \times 0.12 \times 0.04 \mathrm{~mm}^{3}$

1.41 to $26.37^{\circ}$.

$-16<=\mathrm{h}<=16,-19<=\mathrm{k}<=18,0<=\mathrm{l}<=20$

55205

12827 [R(int $)=0.0391]$

$99.7 \%$

Empirical

0.9796 and 0.8685

Full-matrix least-squares on $\mathrm{F}^{2}$

12827 / 0 / 819

1.064

$\mathrm{R} 1=0.0364, \mathrm{wR} 2=0.0925$

$\mathrm{R} 1=0.0505, \mathrm{wR} 2=0.1031$

1.371 and -0.492 e. $\AA^{-3}$ 
Table 2. Atomic coordinates $\left(x 1^{4}\right)$ and equivalent isotropic displacement parameters $\left(\AA^{2} \times 10^{3}\right)$ for $1 b . U(e q)$ is defined as one third of the trace of the orthogonalized $U^{i j}$ tensor.

\begin{tabular}{|c|c|c|c|c|}
\hline & $\mathrm{x}$ & $\mathrm{y}$ & $\mathrm{z}$ & $\mathrm{U}(\mathrm{eq})$ \\
\hline Mo(1) & $480(1)$ & $2397(1)$ & 2558(1) & $13(1)$ \\
\hline $\operatorname{Mo}(2)$ & 5096(1) & 2618(1) & 7452(1) & $14(1)$ \\
\hline $\mathrm{N}(1)$ & $-456(2)$ & $2786(2)$ & 2022(1) & $16(1)$ \\
\hline $\mathrm{N}(1 \mathrm{~A})$ & $4195(2)$ & $2247(2)$ & 7991(1) & $17(1)$ \\
\hline $\mathrm{O}(1)$ & $62(1)$ & 2132(1) & $3548(1)$ & $18(1)$ \\
\hline $\mathrm{O}(2)$ & 1865(1) & 2907(1) & $2544(1)$ & $17(1)$ \\
\hline $\mathrm{O}(3)$ & $510(2)$ & 3890(1) & $3672(1)$ & $20(1)$ \\
\hline $\mathrm{O}(1 \mathrm{~A})$ & 6414(1) & 2050(1) & 7388(1) & $21(1)$ \\
\hline $\mathrm{O}(2 \mathrm{~A})$ & $4770(2)$ & 1095(1) & 6317(1) & $24(1)$ \\
\hline $\mathrm{O}(3 \mathrm{~A})$ & $4516(2)$ & 2914(1) & 6491(1) & $21(1)$ \\
\hline $\mathrm{C}(1)$ & $624(2)$ & $1173(2)$ & 1732(2) & $16(1)$ \\
\hline$C(2)$ & $292(2)$ & $588(2)$ & $862(2)$ & $16(1)$ \\
\hline $\mathrm{C}(3)$ & $752(2)$ & $-264(2)$ & $467(2)$ & $18(1)$ \\
\hline $\mathrm{C}(4)$ & $477(2)$ & $-829(2)$ & $-362(2)$ & $17(1)$ \\
\hline $\mathrm{C}(5)$ & $-1245(2)$ & $3201(2)$ & $1714(2)$ & $18(1)$ \\
\hline $\mathrm{C}(6)$ & $-1016(2)$ & $3693(2)$ & $1172(2)$ & $19(1)$ \\
\hline $\mathrm{C}(7)$ & $-1829(2)$ & $4074(2)$ & $854(2)$ & $24(1)$ \\
\hline $\mathrm{C}(8)$ & $-2818(2)$ & $3979(2)$ & 1062(2) & $26(1)$ \\
\hline $\mathrm{C}(9)$ & $-3029(2)$ & $3513(2)$ & $1617(2)$ & $23(1)$ \\
\hline $\mathrm{C}(10)$ & $-2254(2)$ & $3121(2)$ & 1962(2) & $20(1)$ \\
\hline $\mathrm{C}(11)$ & $223(2)$ & $3198(2)$ & $7(2)$ & $29(1)$ \\
\hline$C(12)$ & $75(2)$ & $3802(2)$ & $938(2)$ & $22(1)$ \\
\hline $\mathrm{C}(13)$ & $391(2)$ & $4809(2)$ & $1110(2)$ & $29(1)$ \\
\hline $\mathrm{C}(14)$ & $-2771(3)$ & $1598(2)$ & 2039(2) & $38(1)$ \\
\hline$C(15)$ & $-2469(2)$ & $2606(2)$ & $2561(2)$ & $22(1)$ \\
\hline $\mathrm{C}(16)$ & $-3285(2)$ & $3043(2)$ & $3187(2)$ & $30(1)$ \\
\hline $\mathrm{C}(17)$ & 2721(2) & $2716(2)$ & 2064(2) & $19(1)$ \\
\hline $\mathrm{C}(18)$ & $2479(2)$ & $2210(2)$ & $1107(2)$ & $31(1)$ \\
\hline$C(19)$ & $3444(2)$ & $2146(2)$ & $2424(2)$ & $28(1)$ \\
\hline $\mathrm{C}(20)$ & $3249(2)$ & $3659(2)$ & 2202(2) & $29(1)$ \\
\hline
\end{tabular}




\begin{tabular}{|c|c|c|c|c|}
\hline $\mathrm{C}(21)$ & $-90(2)$ & 1398(2) & $3837(2)$ & $21(1)$ \\
\hline$C(22)$ & $-566(3)$ & $523(2)$ & $3159(2)$ & $32(1)$ \\
\hline C(23) & $960(2)$ & $1211(2)$ & 4182(2) & $27(1)$ \\
\hline$C(24)$ & $-808(2)$ & $1747(2)$ & $4578(2)$ & $27(1)$ \\
\hline$C(25)$ & 532(3) & $4709(2)$ & $3434(2)$ & $35(1)$ \\
\hline C(26) & 1393(3) & 5334(3) & 3971(3) & $56(1)$ \\
\hline $\mathrm{C}(27)$ & 1461(3) & $5086(2)$ & $4768(3)$ & $44(1)$ \\
\hline$C(28)$ & 1211(3) & $4051(2)$ & $4429(2)$ & $32(1)$ \\
\hline$C(1 \mathrm{~A})$ & $5463(2)$ & $3820(2)$ & $8302(2)$ & $16(1)$ \\
\hline$C(2 A)$ & $5217(2)$ & $4407(2)$ & $9158(2)$ & $16(1)$ \\
\hline$C(3 A)$ & $4431(2)$ & $4176(2)$ & $9605(2)$ & $18(1)$ \\
\hline$C(4 A)$ & $4225(2)$ & $4747(2)$ & $10413(2)$ & $18(1)$ \\
\hline$C(5 A)$ & $3437(2)$ & 1815(2) & $8288(2)$ & $16(1)$ \\
\hline$C(6 A)$ & $3734(2)$ & $1278(2)$ & $8787(2)$ & $18(1)$ \\
\hline$C(7 A)$ & 2959(2) & $857(2)$ & $9080(2)$ & $21(1)$ \\
\hline$C(8 A)$ & $1934(2)$ & $971(2)$ & $8897(2)$ & $23(1)$ \\
\hline$C(9 A)$ & $1654(2)$ & $1491(2)$ & 8395(2) & $21(1)$ \\
\hline$C(10 A)$ & $2395(2)$ & 1922(2) & $8073(2)$ & $19(1)$ \\
\hline $\mathrm{C}(11 \mathrm{~A})$ & $5174(2)$ & $1801(2)$ & $9936(2)$ & $29(1)$ \\
\hline $\mathrm{C}(12 \mathrm{~A})$ & $4857(2)$ & $1177(2)$ & $9011(2)$ & $21(1)$ \\
\hline$C(13 A)$ & $5113(2)$ & $176(2)$ & $8857(2)$ & $29(1)$ \\
\hline$C(14 A)$ & 1809(3) & $3455(2)$ & $8090(2)$ & $33(1)$ \\
\hline$C(15 A)$ & $2102(2)$ & $2484(2)$ & $7525(2)$ & $22(1)$ \\
\hline$C(16 A)$ & $1236(2)$ & $2020(2)$ & $6842(2)$ & $29(1)$ \\
\hline $\mathrm{C}(17 \mathrm{~A})$ & $7389(2)$ & $2177(2)$ & $7807(2)$ & $25(1)$ \\
\hline$C(18 A)$ & $7395(2)$ & 2628(3) & $8778(2)$ & $34(1)$ \\
\hline$C(19 A)$ & $8070(2)$ & $2759(2)$ & $7448(2)$ & $30(1)$ \\
\hline$C(20 A)$ & 7785(3) & 1192(2) & $7560(3)$ & $39(1)$ \\
\hline$C(21 A)$ & $4485(2)$ & $3644(2)$ & $6190(2)$ & $23(1)$ \\
\hline$C(22 A)$ & $4350(3)$ & $4576(2)$ & $6874(2)$ & $30(1)$ \\
\hline$C(23 A)$ & $5500(3)$ & $3653(2)$ & $5759(2)$ & $31(1)$ \\
\hline$C(24 A)$ & $3566(3)$ & $3415(3)$ & $5520(2)$ & $36(1)$ \\
\hline$C(25 A)$ & 4993(3) & $278(2)$ & $6521(2)$ & $37(1)$ \\
\hline$C(26 A)$ & 4023(5) & $-328(3)$ & $6246(3)$ & $72(2)$ \\
\hline$C(27 A)$ & $3590(4)$ & $-121(3)$ & $5507(3)$ & $60(1)$ \\
\hline $\mathrm{C}(28 \mathrm{~A})$ & $3840(3)$ & $900(2)$ & $5751(2)$ & $39(1)$ \\
\hline
\end{tabular}




\begin{tabular}{|c|c|c|c|c|}
\hline $\mathrm{F}(1)$ & $2624(2)$ & $4166(2)$ & $1912(2)$ & $55(1)$ \\
\hline $\mathrm{F}(2)$ & $4112(1)$ & $3582(1)$ & 1781(1) & $32(1)$ \\
\hline F(3) & $3496(2)$ & 4141(1) & 3023(1) & $51(1)$ \\
\hline $\mathrm{F}(4)$ & $3005(2)$ & $1326(2)$ & $2311(2)$ & $59(1)$ \\
\hline $\mathrm{F}(5)$ & $3702(2)$ & 2553(2) & $3248(1)$ & $60(1)$ \\
\hline $\mathrm{F}(6)$ & $4320(1)$ & 1984(1) & $2027(1)$ & $31(1)$ \\
\hline $\mathrm{F}(7)$ & $1616(1)$ & 1019(1) & $3555(1)$ & $35(1)$ \\
\hline $\mathrm{F}(8)$ & $1383(2)$ & 1933(1) & $4828(1)$ & $36(1)$ \\
\hline $\mathrm{F}(9)$ & $931(2)$ & $495(1)$ & $4452(1)$ & $42(1)$ \\
\hline $\mathrm{F}(10)$ & $-1736(2)$ & $1877(2)$ & $4272(1)$ & $44(1)$ \\
\hline $\mathrm{F}(11)$ & $-943(2)$ & $1137(1)$ & $4967(1)$ & $39(1)$ \\
\hline$F(12)$ & $-474(2)$ & 2542(1) & $5172(1)$ & $36(1)$ \\
\hline $\mathrm{F}(1 \mathrm{~A})$ & $7229(2)$ & $695(2)$ & $7904(2)$ & $52(1)$ \\
\hline $\mathrm{F}(2 \mathrm{~A})$ & $8761(2)$ & 1195(2) & $7868(2)$ & $54(1)$ \\
\hline $\mathrm{F}(3 \mathrm{~A})$ & $7740(2)$ & $751(2)$ & $6721(2)$ & $57(1)$ \\
\hline $\mathrm{F}(4 \mathrm{~A})$ & $9032(1)$ & 2889(2) & $7813(2)$ & $46(1)$ \\
\hline $\mathrm{F}(5 \mathrm{~A})$ & 8123(2) & 2397(2) & $6607(2)$ & $54(1)$ \\
\hline $\mathrm{F}(6 \mathrm{~A})$ & 7708(1) & 3601(1) & 7619(1) & $39(1)$ \\
\hline $\mathrm{F}(7 \mathrm{~A})$ & $6285(1)$ & $3756(1)$ & $6335(1)$ & $37(1)$ \\
\hline $\mathrm{F}(8 \mathrm{~A})$ & $5591(2)$ & $4342(2)$ & $5462(1)$ & $45(1)$ \\
\hline $\mathrm{F}(9 \mathrm{~A})$ & $5616(2)$ & 2871(2) & $5107(1)$ & $45(1)$ \\
\hline$F(10 A)$ & $2689(2)$ & $3478(2)$ & $5910(2)$ & $47(1)$ \\
\hline$F(11 A)$ & $3544(2)$ & $4018(2)$ & $5104(2)$ & $51(1)$ \\
\hline$F(12 A)$ & 3573(2) & 2580(1) & 4935(1) & $43(1)$ \\
\hline
\end{tabular}


Table 3. Bond lengths $[\AA ̊]$ and angles $\left[^{\circ}\right]$ for $1 \mathrm{~b}$.

\begin{tabular}{|c|c|}
\hline $\mathrm{Mo}(1)-\mathrm{N}(1)$ & $1.717(2)$ \\
\hline $\mathrm{Mo}(1)-\mathrm{C}(1)$ & 1.931(3) \\
\hline $\mathrm{Mo}(1)-\mathrm{O}(1)$ & $1.9395(18)$ \\
\hline $\mathrm{Mo}(1)-\mathrm{O}(2)$ & $1.9618(18)$ \\
\hline Mo(1)-O(3) & 2.3995(19) \\
\hline $\mathrm{Mo}(2)-\mathrm{N}(1 \mathrm{~A})$ & $1.723(2)$ \\
\hline $\mathrm{Mo}(2)-\mathrm{C}(1 \mathrm{~A})$ & 1.931(3) \\
\hline $\mathrm{Mo}(2)-\mathrm{O}(3 \mathrm{~A})$ & $1.9372(19)$ \\
\hline $\mathrm{Mo}(2)-\mathrm{O}(1 \mathrm{~A})$ & $1.9424(19)$ \\
\hline $\mathrm{Mo}(2)-\mathrm{O}(2 \mathrm{~A})$ & $2.458(2)$ \\
\hline N(1)-C(5) & 1.394(3) \\
\hline $\mathrm{N}(1 \mathrm{~A})-\mathrm{C}(5 \mathrm{~A})$ & $1.390(3)$ \\
\hline $\mathrm{O}(1)-\mathrm{C}(21)$ & $1.395(3)$ \\
\hline $\mathrm{O}(2)-\mathrm{C}(17)$ & $1.388(3)$ \\
\hline $\mathrm{O}(3)-\mathrm{C}(25)$ & $1.454(4)$ \\
\hline $\mathrm{O}(3)-\mathrm{C}(28)$ & $1.463(4)$ \\
\hline $\mathrm{O}(1 \mathrm{~A})-\mathrm{C}(17 \mathrm{~A})$ & 1.394(3) \\
\hline $\mathrm{O}(2 \mathrm{~A})-\mathrm{C}(25 \mathrm{~A})$ & $1.452(4)$ \\
\hline $\mathrm{O}(2 \mathrm{~A})-\mathrm{C}(28 \mathrm{~A})$ & $1.456(4)$ \\
\hline $\mathrm{O}(3 \mathrm{~A})-\mathrm{C}(21 \mathrm{~A})$ & $1.388(3)$ \\
\hline $\mathrm{C}(1)-\mathrm{C}(2)$ & $1.451(4)$ \\
\hline $\mathrm{C}(2)-\mathrm{C}(4) \# 1$ & $1.411(4)$ \\
\hline $\mathrm{C}(2)-\mathrm{C}(3)$ & $1.413(4)$ \\
\hline$C(3)-C(4)$ & $1.373(4)$ \\
\hline $\mathrm{C}(4)-\mathrm{C}(2) \# 1$ & $1.411(4)$ \\
\hline$C(5)-C(6)$ & $1.410(4)$ \\
\hline$C(5)-C(10)$ & $1.419(4)$ \\
\hline$C(6)-C(7)$ & $1.394(4)$ \\
\hline$C(6)-C(12)$ & $1.520(4)$ \\
\hline$C(7)-C(8)$ & $1.374(4)$ \\
\hline C(8)-C(9) & $1.393(4)$ \\
\hline C(9)-C(10) & $1.390(4)$ \\
\hline $\mathrm{C}(10)-\mathrm{C}(15)$ & $1.514(4)$ \\
\hline $\mathrm{C}(11)-\mathrm{C}(12)$ & $1.531(4)$ \\
\hline
\end{tabular}




$\begin{array}{ll}\mathrm{C}(12)-\mathrm{C}(13) & 1.525(4) \\ \mathrm{C}(14)-\mathrm{C}(15) & 1.525(4) \\ \mathrm{C}(15)-\mathrm{C}(16) & 1.529(4) \\ \mathrm{C}(17)-\mathrm{C}(18) & 1.522(4) \\ \mathrm{C}(17)-\mathrm{C}(20) & 1.536(4) \\ \mathrm{C}(17)-\mathrm{C}(19) & 1.542(4) \\ \mathrm{C}(19)-\mathrm{F}(5) & 1.316(4) \\ \mathrm{C}(19)-\mathrm{F}(4) & 1.327(4) \\ \mathrm{C}(19)-\mathrm{F}(6) & 1.338(3) \\ \mathrm{C}(20)-\mathrm{F}(3) & 1.326(4) \\ \mathrm{C}(20)-\mathrm{F}(1) & 1.329(4) \\ \mathrm{C}(20)-\mathrm{F}(2) & 1.347(3) \\ \mathrm{C}(21)-\mathrm{C}(22) & 1.519(4) \\ \mathrm{C}(21)-\mathrm{C}(23) & 1.532(4) \\ \mathrm{C}(21)-\mathrm{C}(24) & 1.543(4) \\ \mathrm{C}(23)-\mathrm{F}(8) & 1.332(4) \\ \mathrm{C}(23)-\mathrm{F}(9) & 1.334(3) \\ \mathrm{C}(23)-\mathrm{F}(7) & 1.345(3) \\ \mathrm{C}(24)-\mathrm{F}(12) & 1.325(4) \\ \mathrm{C}(24)-\mathrm{F}(11) & 1.334(3) \\ \mathrm{C}(24)-\mathrm{F}(10) & 1.337(4) \\ \mathrm{C}(25)-\mathrm{C}(26) & 1.496(6) \\ \mathrm{C}(26)-\mathrm{C}(27) & 1.506(6) \\ \mathrm{C}(27)-\mathrm{C}(28) & 1.520(5) \\ \mathrm{C}(1 \mathrm{~A})-\mathrm{C}(2 \mathrm{~A}) & 1.456(4) \\ \mathrm{C}(2 \mathrm{~A})-\mathrm{C}(3 \mathrm{~A}) & 1.412(4) \\ \mathrm{C}(2 \mathrm{~A})-\mathrm{C}(4 \mathrm{~A}) \# 2 & 1.414(4) \\ \mathrm{C}(3 \mathrm{~A})-\mathrm{C}(4 \mathrm{~A}) & 1.372(4) \\ \mathrm{C}(4 \mathrm{~A})-\mathrm{C}(2 \mathrm{~A}) \# 2 & 1.414(4) \\ \mathrm{C}(5 \mathrm{~A})-\mathrm{C}(6 \mathrm{~A}) & 1.413(4) \\ \mathrm{C}(5 \mathrm{~A})-\mathrm{C}(10 \mathrm{~A}) & 1.418(4) \\ \mathrm{C}(6 \mathrm{~A})-\mathrm{C}(7 \mathrm{~A}) & 1.392(4) \\ \mathrm{C}(6 \mathrm{~A})-\mathrm{C}(12 \mathrm{~A}) & 1.520(4) \\ \mathrm{C}(7 \mathrm{~A})-\mathrm{C}(8 \mathrm{~A}) & 1.387(4) \\ \mathrm{C}(8 \mathrm{~A})-\mathrm{C}(9 \mathrm{~A}) & \\ \mathrm{C}(9 \mathrm{~A})-\mathrm{C}(10 \mathrm{~A}) & \end{array}$




\begin{tabular}{|c|c|}
\hline$C(10 A)-C(15 A)$ & $1.508(4)$ \\
\hline$C(11 A)-C(12 A)$ & $1.534(4)$ \\
\hline$C(12 A)-C(13 A)$ & $1.525(4)$ \\
\hline$C(14 A)-C(15 A)$ & $1.535(4)$ \\
\hline$C(15 A)-C(16 A)$ & $1.530(4)$ \\
\hline$C(17 A)-C(18 A)$ & $1.521(4)$ \\
\hline$C(17 A)-C(19 A)$ & $1.537(4)$ \\
\hline $\mathrm{C}(17 \mathrm{~A})-\mathrm{C}(20 \mathrm{~A})$ & $1.541(4)$ \\
\hline$C(19 A)-F(5 A)$ & $1.321(4)$ \\
\hline$C(19 A)-F(6 A)$ & $1.335(4)$ \\
\hline $\mathrm{C}(19 \mathrm{~A})-\mathrm{F}(4 \mathrm{~A})$ & $1.346(4)$ \\
\hline$C(20 A)-F(3 A)$ & $1.321(5)$ \\
\hline$C(20 A)-F(1 A)$ & $1.334(4)$ \\
\hline$C(20 A)-F(2 A)$ & $1.349(4)$ \\
\hline$C(21 A)-C(22 A)$ & $1.513(4)$ \\
\hline $\mathrm{C}(21 \mathrm{~A})-\mathrm{C}(24 \mathrm{~A})$ & $1.540(4)$ \\
\hline$C(21 A)-C(23 A)$ & $1.545(4)$ \\
\hline C(23A)-F(9A) & $1.330(4)$ \\
\hline $\mathrm{C}(23 \mathrm{~A})-\mathrm{F}(8 \mathrm{~A})$ & $1.331(4)$ \\
\hline C(23A)-F(7A) & $1.334(4)$ \\
\hline $\mathrm{C}(24 \mathrm{~A})-\mathrm{F}(12 \mathrm{~A})$ & $1.317(4)$ \\
\hline $\mathrm{C}(24 \mathrm{~A})-\mathrm{F}(10 \mathrm{~A})$ & $1.336(4)$ \\
\hline $\mathrm{C}(24 \mathrm{~A})-\mathrm{F}(11 \mathrm{~A})$ & $1.346(4)$ \\
\hline$C(25 A)-C(26 A)$ & $1.510(6)$ \\
\hline $\mathrm{C}(26 \mathrm{~A})-\mathrm{C}(27 \mathrm{~A})$ & $1.468(7)$ \\
\hline $\mathrm{C}(27 \mathrm{~A})-\mathrm{C}(28 \mathrm{~A})$ & $1.509(5)$ \\
\hline N(1)-Mo(1)-C(1) & $100.03(11)$ \\
\hline $\mathrm{N}(1)-\mathrm{Mo}(1)-\mathrm{O}(1)$ & $115.83(9)$ \\
\hline $\mathrm{C}(1)-\mathrm{Mo}(1)-\mathrm{O}(1)$ & 102.01(10) \\
\hline $\mathrm{N}(1)-\mathrm{Mo}(1)-\mathrm{O}(2)$ & $114.27(9)$ \\
\hline $\mathrm{C}(1)-\mathrm{Mo}(1)-\mathrm{O}(2)$ & $98.30(9)$ \\
\hline $\mathrm{O}(1)-\mathrm{Mo}(1)-\mathrm{O}(2)$ & $120.81(8)$ \\
\hline N(1)-Mo(1)-O(3) & 87.22(9) \\
\hline $\mathrm{C}(1)-\mathrm{Mo}(1)-\mathrm{O}(3)$ & $172.49(9)$ \\
\hline $\mathrm{O}(1)-\mathrm{Mo}(1)-\mathrm{O}(3)$ & $76.23(7)$ \\
\hline
\end{tabular}




\begin{tabular}{|c|c|}
\hline $\mathrm{O}(2)-\mathrm{Mo}(1)-\mathrm{O}(3)$ & $76.77(7)$ \\
\hline $\mathrm{N}(1 \mathrm{~A})-\mathrm{Mo}(2)-\mathrm{C}(1 \mathrm{~A})$ & $99.27(11)$ \\
\hline $\mathrm{N}(1 \mathrm{~A})-\mathrm{Mo}(2)-\mathrm{O}(3 \mathrm{~A})$ & $113.72(10)$ \\
\hline $\mathrm{C}(1 \mathrm{~A})-\mathrm{Mo}(2)-\mathrm{O}(3 \mathrm{~A})$ & 102.97(10) \\
\hline $\mathrm{N}(1 \mathrm{~A})-\mathrm{Mo}(2)-\mathrm{O}(1 \mathrm{~A})$ & 115.81(9) \\
\hline $\mathrm{C}(1 \mathrm{~A})-\mathrm{Mo}(2)-\mathrm{O}(1 \mathrm{~A})$ & $99.81(10)$ \\
\hline $\mathrm{O}(3 \mathrm{~A})-\mathrm{Mo}(2)-\mathrm{O}(1 \mathrm{~A})$ & $120.34(8)$ \\
\hline N(1A)-Mo(2)-O(2A) & $85.34(9)$ \\
\hline $\mathrm{C}(1 \mathrm{~A})-\mathrm{Mo}(2)-\mathrm{O}(2 \mathrm{~A})$ & $175.07(9)$ \\
\hline $\mathrm{O}(3 \mathrm{~A})-\mathrm{Mo}(2)-\mathrm{O}(2 \mathrm{~A})$ & $76.55(8)$ \\
\hline $\mathrm{O}(1 \mathrm{~A})-\mathrm{Mo}(2)-\mathrm{O}(2 \mathrm{~A})$ & $76.47(8)$ \\
\hline $\mathrm{C}(5)-\mathrm{N}(1)-\mathrm{Mo}(1)$ & 171.01(19) \\
\hline $\mathrm{C}(5 \mathrm{~A})-\mathrm{N}(1 \mathrm{~A})-\mathrm{Mo}(2)$ & 169.66(19) \\
\hline $\mathrm{C}(21)-\mathrm{O}(1)-\mathrm{Mo}(1)$ & $141.46(17)$ \\
\hline $\mathrm{C}(17)-\mathrm{O}(2)-\mathrm{Mo}(1)$ & $140.57(17)$ \\
\hline $\mathrm{C}(25)-\mathrm{O}(3)-\mathrm{C}(28)$ & $109.1(2)$ \\
\hline $\mathrm{C}(25)-\mathrm{O}(3)-\mathrm{Mo}(1)$ & $118.75(17)$ \\
\hline $\mathrm{C}(28)-\mathrm{O}(3)-\mathrm{Mo}(1)$ & $117.64(16)$ \\
\hline $\mathrm{C}(17 \mathrm{~A})-\mathrm{O}(1 \mathrm{~A})-\mathrm{Mo}(2)$ & 141.83(18) \\
\hline $\mathrm{C}(25 \mathrm{~A})-\mathrm{O}(2 \mathrm{~A})-\mathrm{C}(28 \mathrm{~A})$ & $108.5(2)$ \\
\hline $\mathrm{C}(25 \mathrm{~A})-\mathrm{O}(2 \mathrm{~A})-\mathrm{Mo}(2)$ & $118.23(18)$ \\
\hline $\mathrm{C}(28 \mathrm{~A})-\mathrm{O}(2 \mathrm{~A})-\mathrm{Mo}(2)$ & $120.57(18)$ \\
\hline $\mathrm{C}(21 \mathrm{~A})-\mathrm{O}(3 \mathrm{~A})-\mathrm{Mo}(2)$ & $140.20(17)$ \\
\hline $\mathrm{C}(2)-\mathrm{C}(1)-\mathrm{Mo}(1)$ & $141.6(2)$ \\
\hline C(4)\#1-C(2)-C(3) & $116.5(2)$ \\
\hline $\mathrm{C}(4) \# 1-\mathrm{C}(2)-\mathrm{C}(1)$ & $123.1(2)$ \\
\hline $\mathrm{C}(3)-\mathrm{C}(2)-\mathrm{C}(1)$ & $120.4(2)$ \\
\hline $\mathrm{C}(4)-\mathrm{C}(3)-\mathrm{C}(2)$ & 122.3(3) \\
\hline $\mathrm{C}(3)-\mathrm{C}(4)-\mathrm{C}(2) \# 1$ & 121.2(2) \\
\hline $\mathrm{N}(1)-\mathrm{C}(5)-\mathrm{C}(6)$ & 119.1(2) \\
\hline $\mathrm{N}(1)-\mathrm{C}(5)-\mathrm{C}(10)$ & $118.8(2)$ \\
\hline$C(6)-C(5)-C(10)$ & $122.1(2)$ \\
\hline $\mathrm{C}(7)-\mathrm{C}(6)-\mathrm{C}(5)$ & $117.5(3)$ \\
\hline $\mathrm{C}(7)-\mathrm{C}(6)-\mathrm{C}(12)$ & $121.0(3)$ \\
\hline$C(5)-C(6)-C(12)$ & $121.4(2)$ \\
\hline $\mathrm{C}(8)-\mathrm{C}(7)-\mathrm{C}(6)$ & 121.5(3) \\
\hline
\end{tabular}




$\begin{array}{ll}\mathrm{C}(7)-\mathrm{C}(8)-\mathrm{C}(9) & 120.4(3) \\ \mathrm{C}(10)-\mathrm{C}(9)-\mathrm{C}(8) & 121.2(3) \\ \mathrm{C}(9)-\mathrm{C}(10)-\mathrm{C}(5) & 117.3(3) \\ \mathrm{C}(9)-\mathrm{C}(10)-\mathrm{C}(15) & 122.0(3) \\ \mathrm{C}(5)-\mathrm{C}(10)-\mathrm{C}(15) & 120.7(2) \\ \mathrm{C}(6)-\mathrm{C}(12)-\mathrm{C}(13) & 112.1(2) \\ \mathrm{C}(6)-\mathrm{C}(12)-\mathrm{C}(11) & 111.2(2) \\ \mathrm{C}(13)-\mathrm{C}(12)-\mathrm{C}(11) & 111.1(2) \\ \mathrm{C}(10)-\mathrm{C}(15)-\mathrm{C}(14) & 109.6(3) \\ \mathrm{C}(10)-\mathrm{C}(15)-\mathrm{C}(16) & 113.4(2) \\ \mathrm{C}(14)-\mathrm{C}(15)-\mathrm{C}(16) & 110.7(3) \\ \mathrm{O}(2)-\mathrm{C}(17)-\mathrm{C}(18) & 114.3(2) \\ \mathrm{O}(2)-\mathrm{C}(17)-\mathrm{C}(20) & 105.7(2) \\ \mathrm{C}(18)-\mathrm{C}(17)-\mathrm{C}(20) & 109.0(2) \\ \mathrm{O}(2)-\mathrm{C}(17)-\mathrm{C}(19) & 108.6(2) \\ \mathrm{C}(18)-\mathrm{C}(17)-\mathrm{C}(19) & 109.8(2) \\ \mathrm{C}(20)-\mathrm{C}(17)-\mathrm{C}(19) & 109.3(2) \\ \mathrm{F}(5)-\mathrm{C}(19)-\mathrm{F}(4) & 107.7(3) \\ \mathrm{F}(5)-\mathrm{C}(19)-\mathrm{F}(6) & 106.5(3) \\ \mathrm{F}(4)-\mathrm{C}(19)-\mathrm{F}(6) & 106.6(3) \\ \mathrm{F}(5)-\mathrm{C}(19)-\mathrm{C}(17) & 113.1(3) \\ \mathrm{F}(4)-\mathrm{C}(19)-\mathrm{C}(17) & 110.5(3) \\ \mathrm{F}(6)-\mathrm{C}(19)-\mathrm{C}(17) & 112.1(2) \\ \mathrm{F}(3)-\mathrm{C}(20)-\mathrm{F}(1) & 107.1(3) \\ \mathrm{F}(3)-\mathrm{C}(20)-\mathrm{F}(2) & 107.4(3) \\ \mathrm{F}(1)-\mathrm{C}(20)-\mathrm{F}(2) & 107.0(3) \\ \mathrm{F}(3)-\mathrm{C}(20)-\mathrm{C}(17) & 112.7(3) \\ \mathrm{F}(1)-\mathrm{C}(20)-\mathrm{C}(17) & 109.9(3) \\ \mathrm{F}(2)-\mathrm{C}(20)-\mathrm{C}(17) & 112.4(2) \\ \mathrm{O}(1)-\mathrm{C}(21)-\mathrm{C}(22) & 114.4(2) \\ \mathrm{O}(1)-\mathrm{C}(21)-\mathrm{C}(23) & 106.7(2) \\ \mathrm{C}(22)-\mathrm{C}(21)-\mathrm{C}(23) & 110.5(3) \\ \mathrm{O}(1)-\mathrm{C}(21)-\mathrm{C}(24) & 105.8(2) \\ \mathrm{C}(22)-\mathrm{C}(21)-\mathrm{C}(24) & 109.4(2) \\ \mathrm{C}(23)-\mathrm{C}(21)-\mathrm{C}(24) & 109.8(2) \\ \mathrm{F}(8)-\mathrm{C}(23)-\mathrm{F}(9) & 107.3(3)\end{array}$




$\begin{array}{ll}\mathrm{F}(8)-\mathrm{C}(23)-\mathrm{F}(7) & 106.1(3) \\ \mathrm{F}(9)-\mathrm{C}(23)-\mathrm{F}(7) & 107.4(2) \\ \mathrm{F}(8)-\mathrm{C}(23)-\mathrm{C}(21) & 113.5(2) \\ \mathrm{F}(9)-\mathrm{C}(23)-\mathrm{C}(21) & 112.9(3) \\ \mathrm{F}(7)-\mathrm{C}(23)-\mathrm{C}(21) & 109.3(2) \\ \mathrm{F}(12)-\mathrm{C}(24)-\mathrm{F}(11) & 107.7(3) \\ \mathrm{F}(12)-\mathrm{C}(24)-\mathrm{F}(10) & 107.4(3) \\ \mathrm{F}(11)-\mathrm{C}(24)-\mathrm{F}(10) & 106.5(2) \\ \mathrm{F}(12)-\mathrm{C}(24)-\mathrm{C}(21) & 113.0(2) \\ \mathrm{F}(11)-\mathrm{C}(24)-\mathrm{C}(21) & 112.0(2) \\ \mathrm{F}(10)-\mathrm{C}(24)-\mathrm{C}(21) & 109.8(3) \\ \mathrm{O}(3)-\mathrm{C}(25)-\mathrm{C}(26) & 106.7(3) \\ \mathrm{C}(25)-\mathrm{C}(26)-\mathrm{C}(27) & 103.1(3) \\ \mathrm{C}(26)-\mathrm{C}(27)-\mathrm{C}(28) & 103.4(3) \\ \mathrm{O}(3)-\mathrm{C}(28)-\mathrm{C}(27) & 104.8(3) \\ \mathrm{C}(2 \mathrm{~A})-\mathrm{C}(1 \mathrm{~A})-\mathrm{Mo}(2) & 140.4(2) \\ \mathrm{C}(3 \mathrm{~A})-\mathrm{C}(2 \mathrm{~A})-\mathrm{C}(4 \mathrm{~A}) \# 2 & 116.5(2) \\ \mathrm{C}(3 \mathrm{~A})-\mathrm{C}(2 \mathrm{~A})-\mathrm{C}(1 \mathrm{~A}) & 123.1(2) \\ \mathrm{C}(4 \mathrm{~A}) \# 2-\mathrm{C}(2 \mathrm{~A})-\mathrm{C}(1 \mathrm{~A}) & 120.4(2) \\ \mathrm{C}(4 \mathrm{~A})-\mathrm{C}(3 \mathrm{~A})-\mathrm{C}(2 \mathrm{~A}) & 121.3(3) \\ \mathrm{C}(3 \mathrm{~A})-\mathrm{C}(4 \mathrm{~A})-\mathrm{C}(2 \mathrm{~A}) \# 2 & 122.1(3) \\ \mathrm{N}(1 \mathrm{~A})-\mathrm{C}(5 \mathrm{~A})-\mathrm{C}(6 \mathrm{~A}) & 118.8(2) \\ \mathrm{N}(1 \mathrm{~A})-\mathrm{C}(5 \mathrm{~A})-\mathrm{C}(10 \mathrm{~A}) & 118.8(2) \\ \mathrm{C}(6 \mathrm{~A})-\mathrm{C}(5 \mathrm{~A})-\mathrm{C}(10 \mathrm{~A}) & 122.4(2) \\ \mathrm{C}(7 \mathrm{~A})-\mathrm{C}(6 \mathrm{~A})-\mathrm{C}(5 \mathrm{~A}) & 117.6(3) \\ \mathrm{C}(7 \mathrm{~A})-\mathrm{C}(6 \mathrm{~A})-\mathrm{C}(12 \mathrm{~A}) & 121.0(3) \\ \mathrm{C}(5 \mathrm{~A})-\mathrm{C}(6 \mathrm{~A})-\mathrm{C}(12 \mathrm{~A}) & 121.4(2) \\ \mathrm{C}(8 \mathrm{~A})-\mathrm{C}(7 \mathrm{~A})-\mathrm{C}(6 \mathrm{~A}) & 121.0(3) \\ \mathrm{C}(7 \mathrm{~A})-\mathrm{C}(8 \mathrm{~A})-\mathrm{C}(9 \mathrm{~A}) & 120.7(3) \\ \mathrm{C}(8 \mathrm{~A})-\mathrm{C}(9 \mathrm{~A})-\mathrm{C}(10 \mathrm{~A}) & 121.0(3) \\ \mathrm{C}(9 \mathrm{~A})-\mathrm{C}(10 \mathrm{~A})-\mathrm{C}(5 \mathrm{~A}) & 117.2(3) \\ \mathrm{C}(9 \mathrm{~A})-\mathrm{C}(10 \mathrm{~A})-\mathrm{C}(15 \mathrm{~A}) & 121.6(3) \\ \mathrm{C}(5 \mathrm{~A})-\mathrm{C}(10 \mathrm{~A})-\mathrm{C}(15 \mathrm{~A}) & 121.2(2) \\ \mathrm{C}(6 \mathrm{~A})-\mathrm{C}(12 \mathrm{~A})-\mathrm{C}(13 \mathrm{~A}) & 112.6(2) \\ \mathrm{C}(6 \mathrm{~A})-\mathrm{C}(12 \mathrm{~A})-\mathrm{C}(11 \mathrm{~A}) & 110.9(2) \\ \mathrm{C}(13 \mathrm{~A})-\mathrm{C}(12 \mathrm{~A})-\mathrm{C}(11 \mathrm{~A}) & 111.0(2)\end{array}$




$$
\begin{array}{ll}
\mathrm{C}(10 \mathrm{~A})-\mathrm{C}(15 \mathrm{~A})-\mathrm{C}(16 \mathrm{~A}) & 113.1(2) \\
\mathrm{C}(10 \mathrm{~A})-\mathrm{C}(15 \mathrm{~A})-\mathrm{C}(14 \mathrm{~A}) & 110.3(2) \\
\mathrm{C}(16 \mathrm{~A})-\mathrm{C}(15 \mathrm{~A})-\mathrm{C}(14 \mathrm{~A}) & 110.3(2) \\
\mathrm{O}(1 \mathrm{~A})-\mathrm{C}(17 \mathrm{~A})-\mathrm{C}(18 \mathrm{~A}) & 114.0(2) \\
\mathrm{O}(1 \mathrm{~A})-\mathrm{C}(17 \mathrm{~A})-\mathrm{C}(19 \mathrm{~A}) & 108.8(2) \\
\mathrm{C}(18 \mathrm{~A})-\mathrm{C}(17 \mathrm{~A})-\mathrm{C}(19 \mathrm{~A}) & 109.8(3) \\
\mathrm{O}(1 \mathrm{~A})-\mathrm{C}(17 \mathrm{~A})-\mathrm{C}(20 \mathrm{~A}) & 104.1(3) \\
\mathrm{C}(18 \mathrm{~A})-\mathrm{C}(17 \mathrm{~A})-\mathrm{C}(20 \mathrm{~A}) & 110.3(3) \\
\mathrm{C}(19 \mathrm{~A})-\mathrm{C}(17 \mathrm{~A})-\mathrm{C}(20 \mathrm{~A}) & 109.6(3) \\
\mathrm{F}(5 \mathrm{~A})-\mathrm{C}(19 \mathrm{~A})-\mathrm{F}(6 \mathrm{~A}) & 106.8(3) \\
\mathrm{F}(5 \mathrm{~A})-\mathrm{C}(19 \mathrm{~A})-\mathrm{F}(4 \mathrm{~A}) & 108.1(3) \\
\mathrm{F}(6 \mathrm{~A})-\mathrm{C}(19 \mathrm{~A})-\mathrm{F}(4 \mathrm{~A}) & 106.0(3) \\
\mathrm{F}(5 \mathrm{~A})-\mathrm{C}(19 \mathrm{~A})-\mathrm{C}(17 \mathrm{~A}) & 113.3(3) \\
\mathrm{F}(6 \mathrm{~A})-\mathrm{C}(19 \mathrm{~A})-\mathrm{C}(17 \mathrm{~A}) & 110.5(2) \\
\mathrm{F}(4 \mathrm{~A})-\mathrm{C}(19 \mathrm{~A})-\mathrm{C}(17 \mathrm{~A}) & 111.7(3) \\
\mathrm{F}(3 \mathrm{~A})-\mathrm{C}(20 \mathrm{~A})-\mathrm{F}(1 \mathrm{~A}) & 107.9(3) \\
\mathrm{F}(3 \mathrm{~A})-\mathrm{C}(20 \mathrm{~A})-\mathrm{F}(2 \mathrm{~A}) & 108.2(3) \\
\mathrm{F}(1 \mathrm{~A})-\mathrm{C}(20 \mathrm{~A})-\mathrm{F}(2 \mathrm{~A}) & 105.9(3) \\
\mathrm{F}(3 \mathrm{~A})-\mathrm{C}(20 \mathrm{~A})-\mathrm{C}(17 \mathrm{~A}) & 112.6(3) \\
\mathrm{F}(1 \mathrm{~A})-\mathrm{C}(20 \mathrm{~A})-\mathrm{C}(17 \mathrm{~A}) & 110.1(3) \\
\mathrm{F}(2 \mathrm{~A})-\mathrm{C}(20 \mathrm{~A})-\mathrm{C}(17 \mathrm{~A}) & 111.9(3) \\
\mathrm{O}(3 \mathrm{~A})-\mathrm{C}(21 \mathrm{~A})-\mathrm{C}(22 \mathrm{~A}) & 114.3(2) \\
\mathrm{O}(3 \mathrm{~A})-\mathrm{C}(21 \mathrm{~A})-\mathrm{C}(24 \mathrm{~A}) & 105.9(2) \\
\mathrm{C}(22 \mathrm{~A})-\mathrm{C}(21 \mathrm{~A})-\mathrm{C}(24 \mathrm{~A}) & 109.2(3) \\
\mathrm{O}(3 \mathrm{~A})-\mathrm{C}(21 \mathrm{~A})-\mathrm{C}(23 \mathrm{~A}) & 106.6(2) \\
\mathrm{C}(22 \mathrm{~A})-\mathrm{C}(21 \mathrm{~A})-\mathrm{C}(23 \mathrm{~A}) & 110.2(3) \\
\mathrm{C}(24 \mathrm{~A})-\mathrm{C}(21 \mathrm{~A})-\mathrm{C}(23 \mathrm{~A}) & 110.6(3) \\
\mathrm{F}(9 \mathrm{~A})-\mathrm{C}(23 \mathrm{~A})-\mathrm{F}(8 \mathrm{~A}) & 107.2(3) \\
\mathrm{F}(9 \mathrm{~A})-\mathrm{C}(23 \mathrm{~A})-\mathrm{F}(7 \mathrm{~A}) & 107.5(3) \\
\mathrm{F}(8 \mathrm{~A})-\mathrm{C}(23 \mathrm{~A})-\mathrm{F}(7 \mathrm{~A}) & 107.1(3) \\
\mathrm{F}(9 \mathrm{~A})-\mathrm{C}(23 \mathrm{~A})-\mathrm{C}(21 \mathrm{~A}) & 112.4(3) \\
\mathrm{F}(8 \mathrm{~A})-\mathrm{C}(23 \mathrm{~A})-\mathrm{C}(21 \mathrm{~A}) & 113.2(3) \\
\mathrm{F}(7 \mathrm{~A})-\mathrm{C}(23 \mathrm{~A})-\mathrm{C}(21 \mathrm{~A}) & 109.1(3) \\
\mathrm{F}(12 \mathrm{~A})-\mathrm{C}(24 \mathrm{~A})-\mathrm{F}(10 \mathrm{~A}) & 107.7(3) \\
\mathrm{F}(12 \mathrm{~A})-\mathrm{C}(24 \mathrm{~A})-\mathrm{F}(11 \mathrm{~A}) & 107.2(3) \\
\mathrm{F}(10 \mathrm{~A})-\mathrm{C}(24 \mathrm{~A})-\mathrm{F}(11 \mathrm{~A}) & 107.3(3)
\end{array}
$$


$\mathrm{F}(12 \mathrm{~A})-\mathrm{C}(24 \mathrm{~A})-\mathrm{C}(21 \mathrm{~A}) \quad 113.6(3)$

$\mathrm{F}(10 \mathrm{~A})-\mathrm{C}(24 \mathrm{~A})-\mathrm{C}(21 \mathrm{~A}) \quad 110.0(3)$

$\mathrm{F}(11 \mathrm{~A})-\mathrm{C}(24 \mathrm{~A})-\mathrm{C}(21 \mathrm{~A}) \quad 110.8(3)$

$\mathrm{O}(2 \mathrm{~A})-\mathrm{C}(25 \mathrm{~A})-\mathrm{C}(26 \mathrm{~A}) \quad 105.0(3)$

$\mathrm{C}(27 \mathrm{~A})-\mathrm{C}(26 \mathrm{~A})-\mathrm{C}(25 \mathrm{~A}) \quad 103.7(3)$

$\mathrm{C}(26 \mathrm{~A})-\mathrm{C}(27 \mathrm{~A})-\mathrm{C}(28 \mathrm{~A}) \quad 103.3(3)$

$\mathrm{O}(2 \mathrm{~A})-\mathrm{C}(28 \mathrm{~A})-\mathrm{C}(27 \mathrm{~A}) \quad 105.9(3)$

Symmetry transformations used to generate equivalent atoms:

$\# 1-x,-y,-z \quad \# 2-x+1,-y+1,-z+2$ 
Table 4. Anisotropic displacement parameters $\left(\AA^{2} \times 10^{3}\right)$ for $1 \mathrm{~b}$. The anisotropic displacement factor exponent takes the form: $-2 \pi^{2}\left[h^{2} a^{* 2} U^{11}+\ldots+2 h \mathbf{k}^{*} \mathbf{b}^{*} \mathbf{U}^{12}\right]$

\begin{tabular}{lcccccc}
\hline & $\mathrm{U} 11$ & $\mathrm{U}^{22}$ & $\mathrm{U}^{33}$ & $\mathrm{U}^{23}$ & $\mathrm{U}^{13}$ & $\mathrm{U} 12$ \\
\hline $\mathrm{Mo}(1)$ & $12(1)$ & $14(1)$ & $15(1)$ & $6(1)$ & $1(1)$ & $0(1)$ \\
$\mathrm{Mo}(2)$ & $14(1)$ & $14(1)$ & $15(1)$ & $5(1)$ & $3(1)$ & $0(1)$ \\
$\mathrm{N}(1)$ & $16(1)$ & $15(1)$ & $17(1)$ & $5(1)$ & $3(1)$ & $1(1)$ \\
$\mathrm{N}(1 \mathrm{~A})$ & $18(1)$ & $15(1)$ & $16(1)$ & $4(1)$ & $1(1)$ & $1(1)$ \\
$\mathrm{O}(1)$ & $21(1)$ & $17(1)$ & $18(1)$ & $8(1)$ & $3(1)$ & $-1(1)$ \\
$\mathrm{O}(2)$ & $14(1)$ & $17(1)$ & $20(1)$ & $4(1)$ & $4(1)$ & $0(1)$ \\
$\mathrm{O}(3)$ & $21(1)$ & $17(1)$ & $20(1)$ & $7(1)$ & $1(1)$ & $0(1)$ \\
$\mathrm{O}(1 \mathrm{~A})$ & $16(1)$ & $21(1)$ & $24(1)$ & $6(1)$ & $3(1)$ & $3(1)$ \\
$\mathrm{O}(2 \mathrm{~A})$ & $29(1)$ & $19(1)$ & $23(1)$ & $5(1)$ & $3(1)$ & $-2(1)$ \\
$\mathrm{O}(3 \mathrm{~A})$ & $27(1)$ & $20(1)$ & $18(1)$ & $9(1)$ & $-2(1)$ & $-2(1)$ \\
$\mathrm{C}(1)$ & $16(1)$ & $17(1)$ & $19(1)$ & $10(1)$ & $2(1)$ & $2(1)$ \\
$\mathrm{C}(2)$ & $14(1)$ & $16(1)$ & $19(1)$ & $7(1)$ & $2(1)$ & $-3(1)$ \\
$\mathrm{C}(3)$ & $16(1)$ & $18(1)$ & $21(1)$ & $8(1)$ & $-1(1)$ & $1(1)$ \\
$\mathrm{C}(4)$ & $17(1)$ & $13(1)$ & $21(1)$ & $5(1)$ & $2(1)$ & $2(1)$ \\
$\mathrm{C}(5)$ & $16(1)$ & $17(1)$ & $20(1)$ & $6(1)$ & $-2(1)$ & $1(1)$ \\
$\mathrm{C}(6)$ & $19(1)$ & $15(1)$ & $22(1)$ & $6(1)$ & $1(1)$ & $2(1)$ \\
$\mathrm{C}(7)$ & $29(2)$ & $21(1)$ & $25(2)$ & $12(1)$ & $-1(1)$ & $4(1)$ \\
$\mathrm{C}(8)$ & $25(2)$ & $24(2)$ & $29(2)$ & $10(1)$ & $-4(1)$ & $8(1)$ \\
$\mathrm{C}(9)$ & $16(1)$ & $22(1)$ & $31(2)$ & $8(1)$ & $0(1)$ & $3(1)$ \\
$\mathrm{C}(10)$ & $18(1)$ & $16(1)$ & $23(1)$ & $6(1)$ & $1(1)$ & $2(1)$ \\
$\mathrm{C}(11)$ & $26(2)$ & $33(2)$ & $30(2)$ & $13(1)$ & $8(1)$ & $2(1)$ \\
$\mathrm{C}(12)$ & $22(1)$ & $22(1)$ & $26(2)$ & $14(1)$ & $2(1)$ & $1(1)$ \\
$\mathrm{C}(13)$ & $28(2)$ & $29(2)$ & $34(2)$ & $17(1)$ & $1(1)$ & $-3(1)$ \\
$\mathrm{C}(14)$ & $48(2)$ & $24(2)$ & $43(2)$ & $12(2)$ & $14(2)$ & $-3(2)$ \\
$\mathrm{C}(15)$ & $17(1)$ & $23(1)$ & $30(2)$ & $13(1)$ & $5(1)$ & $2(1)$ \\
$\mathrm{C}(16)$ & $24(2)$ & $37(2)$ & $30(2)$ & $13(1)$ & $6(1)$ & $6(1)$ \\
$\mathrm{C}(17)$ & $14(1)$ & $21(1)$ & $19(1)$ & $5(1)$ & $4(1)$ & $1(1)$ \\
$\mathrm{C}(18)$ & $19(2)$ & $47(2)$ & $22(2)$ & $5(1)$ & $5(1)$ & $-3(1)$ \\
$\mathrm{C}(19)$ & $20(2)$ & $31(2)$ & $37(2)$ & $17(1)$ & $8(1)$ & $7(1)$ \\
$\mathrm{C}(20)$ & $21(2)$ & $26(2)$ & $43(2)$ & $13(1)$ & $12(1)$ & $3(1)$ \\
$\mathrm{C}(21)$ & $25(1)$ & $18(1)$ & $22(1)$ & $10(1)$ & $5(1)$ & $-1(1)$ \\
& & & & & &
\end{tabular}




\begin{tabular}{|c|c|c|c|c|c|c|}
\hline$C(22)$ & $43(2)$ & $25(2)$ & $28(2)$ & $9(1)$ & $3(1)$ & $-11(1)$ \\
\hline C(23) & $33(2)$ & $26(2)$ & $26(2)$ & $13(1)$ & $8(1)$ & $7(1)$ \\
\hline$C(24)$ & $30(2)$ & $26(2)$ & $31(2)$ & $16(1)$ & $10(1)$ & $3(1)$ \\
\hline$C(25)$ & $60(2)$ & $17(2)$ & $30(2)$ & $10(1)$ & $13(2)$ & $5(1)$ \\
\hline$C(26)$ & $62(3)$ & $22(2)$ & $76(3)$ & $3(2)$ & $32(2)$ & $-7(2)$ \\
\hline $\mathrm{C}(27)$ & $31(2)$ & $32(2)$ & $54(2)$ & $-4(2)$ & $-4(2)$ & $-2(2)$ \\
\hline$C(28)$ & $30(2)$ & $31(2)$ & $27(2)$ & $1(1)$ & $-8(1)$ & $4(1)$ \\
\hline $\mathrm{C}(1 \mathrm{~A})$ & $14(1)$ & $17(1)$ & $19(1)$ & $9(1)$ & $2(1)$ & $1(1)$ \\
\hline$C(2 A)$ & $15(1)$ & $17(1)$ & $18(1)$ & $8(1)$ & $-1(1)$ & $3(1)$ \\
\hline $\mathrm{C}(3 \mathrm{~A})$ & $18(1)$ & $15(1)$ & $22(1)$ & $6(1)$ & $1(1)$ & $-2(1)$ \\
\hline$C(4 A)$ & $16(1)$ & $18(1)$ & $21(1)$ & $8(1)$ & $3(1)$ & $-2(1)$ \\
\hline$C(5 A)$ & $19(1)$ & $11(1)$ & $16(1)$ & $3(1)$ & $5(1)$ & $-1(1)$ \\
\hline$C(6 A)$ & $22(1)$ & $15(1)$ & $18(1)$ & $5(1)$ & $5(1)$ & $2(1)$ \\
\hline$C(7 A)$ & $30(2)$ & $17(1)$ & $19(1)$ & $8(1)$ & $5(1)$ & $1(1)$ \\
\hline$C(8 A)$ & $24(2)$ & $23(1)$ & $22(1)$ & $6(1)$ & $7(1)$ & $-5(1)$ \\
\hline $\mathrm{C}(9 \mathrm{~A})$ & $17(1)$ & $22(1)$ & $23(1)$ & $5(1)$ & $4(1)$ & $-2(1)$ \\
\hline$C(10 A)$ & 21(1) & $15(1)$ & $17(1)$ & $2(1)$ & $3(1)$ & $0(1)$ \\
\hline$C(11 A)$ & $25(2)$ & $31(2)$ & $28(2)$ & $9(1)$ & $-2(1)$ & $3(1)$ \\
\hline$C(12 A)$ & $23(1)$ & $22(1)$ & $22(1)$ & 11(1) & $2(1)$ & $3(1)$ \\
\hline$C(13 A)$ & $31(2)$ & $26(2)$ & $34(2)$ & $15(1)$ & $5(1)$ & $8(1)$ \\
\hline$C(14 A)$ & $42(2)$ & $25(2)$ & $34(2)$ & $11(1)$ & $-2(2)$ & $9(1)$ \\
\hline$C(15 A)$ & 17(1) & $26(2)$ & $25(2)$ & $12(1)$ & $3(1)$ & $1(1)$ \\
\hline$C(16 A)$ & $25(2)$ & $38(2)$ & $26(2)$ & $15(1)$ & $-3(1)$ & $-1(1)$ \\
\hline$C(17 A)$ & 18(1) & $28(2)$ & $31(2)$ & $12(1)$ & $3(1)$ & $5(1)$ \\
\hline$C(18 A)$ & $22(2)$ & $49(2)$ & $30(2)$ & $13(2)$ & $-3(1)$ & $3(1)$ \\
\hline$C(19 A)$ & $20(2)$ & $32(2)$ & $39(2)$ & $14(1)$ & $7(1)$ & $1(1)$ \\
\hline$C(20 A)$ & $28(2)$ & $35(2)$ & $61(2)$ & $25(2)$ & $9(2)$ & $9(1)$ \\
\hline$C(21 A)$ & $23(2)$ & $22(1)$ & $25(2)$ & $12(1)$ & $-3(1)$ & $-4(1)$ \\
\hline$C(22 A)$ & $35(2)$ & $25(2)$ & $34(2)$ & $13(1)$ & $-1(1)$ & $5(1)$ \\
\hline$C(23 A)$ & 33(2) & $32(2)$ & $31(2)$ & $15(1)$ & $4(1)$ & $-5(1)$ \\
\hline$C(24 A)$ & $35(2)$ & $42(2)$ & $36(2)$ & $22(2)$ & $-10(2)$ & $-7(2)$ \\
\hline$C(25 A)$ & $65(2)$ & $17(2)$ & $28(2)$ & $7(1)$ & $6(2)$ & $3(2)$ \\
\hline$C(26 A)$ & $112(4)$ & $40(2)$ & $60(3)$ & $15(2)$ & $-2(3)$ & $-36(3)$ \\
\hline$C(27 A)$ & 63(3) & $46(2)$ & $61(3)$ & $8(2)$ & $-1(2)$ & $-25(2)$ \\
\hline$C(28 A)$ & $28(2)$ & $33(2)$ & $40(2)$ & $-5(2)$ & $-1(1)$ & $-4(1)$ \\
\hline $\mathrm{F}(1)$ & $32(1)$ & $45(1)$ & $112(2)$ & $55(1)$ & $26(1)$ & $12(1)$ \\
\hline
\end{tabular}




\begin{tabular}{llllccc}
$\mathrm{F}(2)$ & $21(1)$ & $31(1)$ & $47(1)$ & $16(1)$ & $14(1)$ & $-1(1)$ \\
$\mathrm{F}(3)$ & $46(1)$ & $38(1)$ & $48(1)$ & $-12(1)$ & $16(1)$ & $-24(1)$ \\
$\mathrm{F}(4)$ & $26(1)$ & $45(1)$ & $128(2)$ & $58(2)$ & $16(1)$ & $8(1)$ \\
$\mathrm{F}(5)$ & $57(2)$ & $97(2)$ & $30(1)$ & $24(1)$ & $2(1)$ & $37(1)$ \\
$\mathrm{F}(6)$ & $16(1)$ & $34(1)$ & $47(1)$ & $17(1)$ & $9(1)$ & $6(1)$ \\
$\mathrm{F}(7)$ & $32(1)$ & $44(1)$ & $35(1)$ & $21(1)$ & $14(1)$ & $16(1)$ \\
$\mathrm{F}(8)$ & $34(1)$ & $41(1)$ & $29(1)$ & $10(1)$ & $-6(1)$ & $1(1)$ \\
$\mathrm{F}(9)$ & $55(1)$ & $38(1)$ & $48(1)$ & $31(1)$ & $15(1)$ & $19(1)$ \\
$\mathrm{F}(10)$ & $24(1)$ & $69(2)$ & $51(1)$ & $33(1)$ & $13(1)$ & $10(1)$ \\
$\mathrm{F}(11)$ & $48(1)$ & $38(1)$ & $41(1)$ & $25(1)$ & $21(1)$ & $2(1)$ \\
$\mathrm{F}(12)$ & $49(1)$ & $28(1)$ & $29(1)$ & $7(1)$ & $17(1)$ & $5(1)$ \\
$\mathrm{F}(1 \mathrm{~A})$ & $37(1)$ & $40(1)$ & $96(2)$ & $44(1)$ & $10(1)$ & $8(1)$ \\
$\mathrm{F}(2 \mathrm{~A})$ & $25(1)$ & $56(1)$ & $96(2)$ & $45(1)$ & $8(1)$ & $17(1)$ \\
$\mathrm{F}(3 \mathrm{~A})$ & $62(2)$ & $36(1)$ & $63(2)$ & $2(1)$ & $18(1)$ & $25(1)$ \\
$\mathrm{F}(4 \mathrm{~A})$ & $15(1)$ & $47(1)$ & $81(2)$ & $30(1)$ & $2(1)$ & $3(1)$ \\
$\mathrm{F}(5 \mathrm{~A})$ & $57(1)$ & $59(2)$ & $46(1)$ & $16(1)$ & $28(1)$ & $-5(1)$ \\
$\mathrm{F}(6 \mathrm{~A})$ & $23(1)$ & $33(1)$ & $67(1)$ & $25(1)$ & $7(1)$ & $1(1)$ \\
$\mathrm{F}(7 \mathrm{~A})$ & $23(1)$ & $45(1)$ & $46(1)$ & $22(1)$ & $0(1)$ & $-4(1)$ \\
$\mathrm{F}(8 \mathrm{~A})$ & $47(1)$ & $51(1)$ & $53(1)$ & $37(1)$ & $3(1)$ & $-13(1)$ \\
$\mathrm{F}(9 \mathrm{~A})$ & $51(1)$ & $46(1)$ & $35(1)$ & $9(1)$ & $17(1)$ & $-3(1)$ \\
$\mathrm{F}(10 \mathrm{~A})$ & $23(1)$ & $64(2)$ & $57(1)$ & $26(1)$ & $-8(1)$ & $-5(1)$ \\
$\mathrm{F}(11 \mathrm{~A})$ & $54(1)$ & $57(1)$ & $54(1)$ & $41(1)$ & $-24(1)$ & $-11(1)$ \\
$\mathrm{F}(12 \mathrm{~A})$ & $53(1)$ & $45(1)$ & $29(1)$ & $14(1)$ & $-15(1)$ & $-15(1)$ \\
& & & & & & \\
\hline
\end{tabular}


Table 5. Crystal data and structure refinement for 3.

Empirical formula

Formula weight

Temperature

Wavelength

Crystal system

Space group

Unit cell dimensions

Volume

Z

Density (calculated)

Absorption coefficient

$\mathrm{F}(000)$

Crystal size

Theta range for data collection

Index ranges

Reflections collected

Independent reflections

Completeness to theta $=28.33^{\circ}$

Absorption correction

Refinement method

Data / restraints / parameters

Goodness-of-fit on $\mathrm{F}^{2}$

Final $\mathrm{R}$ indices [I>2sigma(I)]

$\mathrm{R}$ indices (all data)

Largest diff. peak and hole
C58 H62 F24 Fe Mo2 N2 O4

1554.83

194(2) K

$0.71073 \AA$

Triclinic

P1

$$
\begin{array}{ll}
\mathrm{a}=10.6901(11) \AA & \alpha=101.756(2)^{\circ} . \\
\mathrm{b}=11.1070(12) \AA & \beta=103.821(2)^{\circ} . \\
\mathrm{c}=15.2513(17) \AA & \gamma=106.968(2)^{\circ} .
\end{array}
$$

1607.3(3) $\AA^{3}$

1

$1.606 \mathrm{Mg} / \mathrm{m}^{3}$

$0.722 \mathrm{~mm}^{-1}$

782

$0.22 \times 0.17 \times 0.06 \mathrm{~mm}^{3}$

2.01 to $28.33^{\circ}$.

$-14<=\mathrm{h}<=14,-14<=\mathrm{k}<=14,-18<=\mathrm{l}<=20$

27210

$7977[\mathrm{R}($ int $)=0.0264]$

$99.4 \%$

Empirical

Full-matrix least-squares on $\mathrm{F}^{2}$

7977 / 0 / 416

1.024

$\mathrm{R} 1=0.0297, \mathrm{wR} 2=0.0757$

$\mathrm{R} 1=0.0342, \mathrm{wR} 2=0.0787$

0.536 and -0.298 e. $\AA^{-3}$ 
Table 6. Atomic coordinates $\left(x^{1} 0^{4}\right)$ and equivalent isotropic displacement parameters $\left(\AA^{2} \times 1^{3}\right)$ for 3 . $U(e q)$ is defined as one third of the trace of the orthogonalized $U^{i j}$ tensor.

\begin{tabular}{|c|c|c|c|c|}
\hline & $\mathrm{x}$ & $\mathrm{y}$ & $\mathrm{z}$ & $\mathrm{U}(\mathrm{eq})$ \\
\hline Mo(1) & $3518(1)$ & 2491(1) & 2213(1) & $23(1)$ \\
\hline $\mathrm{Fe}(1)$ & 5000 & 0 & 0 & $24(1)$ \\
\hline $\mathrm{N}(1)$ & $5267(2)$ & 3362(1) & 2573(1) & $26(1)$ \\
\hline $\mathrm{O}(1)$ & 2484(1) & $3628(1)$ & 2001(1) & $31(1)$ \\
\hline $\mathrm{O}(2)$ & 2783(1) & 1302(1) & 2885(1) & $33(1)$ \\
\hline $\mathrm{C}(1)$ & $3309(2)$ & $1457(2)$ & 986(1) & $27(1)$ \\
\hline $\mathrm{F}(7)$ & $63(2)$ & $1698(2)$ & 838(1) & $67(1)$ \\
\hline$F(5)$ & $781(2)$ & $-635(2)$ & 1314(1) & $84(1)$ \\
\hline$C(8)$ & $7570(2)$ & $3522(2)$ & 3389(1) & $31(1)$ \\
\hline $\mathrm{C}(7)$ & $6676(2)$ & $4102(2)$ & 2954(1) & $27(1)$ \\
\hline C(3) & $5559(2)$ & $2007(2)$ & $527(1)$ & $30(1)$ \\
\hline $\mathrm{C}(13)$ & $7011(2)$ & $2140(2)$ & $3460(2)$ & $38(1)$ \\
\hline $\mathrm{F}(11)$ & $3003(2)$ & 6135(1) & $1775(1)$ & $72(1)$ \\
\hline $\mathrm{F}(12)$ & $1591(2)$ & $5594(2)$ & 2523(1) & $67(1)$ \\
\hline $\mathrm{C}(6)$ & $6477(2)$ & $-653(2)$ & $650(1)$ & $30(1)$ \\
\hline$C(2)$ & $4105(2)$ & $1381(2)$ & $340(1)$ & $27(1)$ \\
\hline$C(23)$ & $1609(2)$ & $3881(2)$ & $1287(1)$ & $33(1)$ \\
\hline $\mathrm{C}(12)$ & $7148(2)$ & $5397(2)$ & 2888(1) & $34(1)$ \\
\hline $\mathrm{F}(9)$ & $-269(2)$ & $3091(2)$ & 1860(1) & $77(1)$ \\
\hline$C(5)$ & $5400(2)$ & $-854(2)$ & 1060(1) & $33(1)$ \\
\hline C(19) & $2207(2)$ & $14(2)$ & 2892(2) & $34(1)$ \\
\hline$C(16)$ & $6176(2)$ & $5972(2)$ & $2378(2)$ & $39(1)$ \\
\hline$F(3)$ & $1545(2)$ & $-1108(2)$ & 3979(1) & $88(1)$ \\
\hline $\mathrm{F}(1)$ & $3379(2)$ & $621(2)$ & $4524(1)$ & $81(1)$ \\
\hline$C(25)$ & $138(2)$ & 2950(3) & $1106(2)$ & $50(1)$ \\
\hline$C(4)$ & $4148(2)$ & $-1691(2)$ & 335(2) & $34(1)$ \\
\hline $\mathrm{F}(10)$ & $847(2)$ & $5667(2)$ & 1110(1) & 81(1) \\
\hline$F(2)$ & $1422(2)$ & $827(2)$ & $4151(1)$ & $86(1)$ \\
\hline $\mathrm{F}(4)$ & $128(2)$ & $-1868(2)$ & $2146(1)$ & $78(1)$ \\
\hline $\mathrm{C}(15)$ & $6504(2)$ & 2171(2) & 4322(2) & $49(1)$ \\
\hline
\end{tabular}




\begin{tabular}{lrrrr}
$\mathrm{F}(6)$ & $-72(2)$ & $34(2)$ & $2351(2)$ & $94(1)$ \\
$\mathrm{C}(9)$ & $8968(2)$ & $4295(2)$ & $3772(2)$ & $42(1)$ \\
$\mathrm{C}(11)$ & $8557(2)$ & $6113(2)$ & $3289(2)$ & $46(1)$ \\
$\mathrm{C}(10)$ & $9449(2)$ & $5571(3)$ & $3724(2)$ & $49(1)$ \\
$\mathrm{F}(8)$ & $-781(2)$ & $3096(2)$ & $409(2)$ & $95(1)$ \\
$\mathrm{C}(24)$ & $1962(2)$ & $3711(2)$ & $367(2)$ & $44(1)$ \\
$\mathrm{C}(26)$ & $1750(3)$ & $5327(2)$ & $1676(2)$ & $48(1)$ \\
$\mathrm{C}(22)$ & $748(3)$ & $-617(3)$ & $2177(2)$ & $53(1)$ \\
$\mathrm{C}(21)$ & $2120(3)$ & $95(3)$ & $3896(2)$ & $56(1)$ \\
$\mathrm{C}(17)$ & $6125(3)$ & $5725(3)$ & $1344(2)$ & $60(1)$ \\
$\mathrm{C}(14)$ & $8044(3)$ & $1431(3)$ & $3485(2)$ & $66(1)$ \\
$\mathrm{C}(20)$ & $3085(3)$ & $-791(3)$ & $2668(3)$ & $71(1)$ \\
$\mathrm{C}(28)$ & $3972(4)$ & $4037(3)$ & $5110(2)$ & $77(1)$ \\
$\mathrm{C}(29)$ & $3668(4)$ & $4592(3)$ & $4394(2)$ & $72(1)$ \\
$\mathrm{C}(18)$ & $6544(3)$ & $7448(2)$ & $2836(2)$ & $57(1)$ \\
$\mathrm{C}(27)$ & $5295(4)$ & $4441(3)$ & $5709(2)$ & $77(1)$ \\
& & & & \\
\hline
\end{tabular}


Table 7. Bond lengths $[\AA ̊]$ and angles $\left[{ }^{\circ}\right]$ for 3 .

\begin{tabular}{|c|c|}
\hline $\mathrm{Mo}(1)-\mathrm{N}(1)$ & $1.7241(15)$ \\
\hline $\mathrm{Mo}(1)-\mathrm{C}(1)$ & 1.9098(18) \\
\hline $\mathrm{Mo}(1)-\mathrm{O}(1)$ & $1.9309(12)$ \\
\hline $\mathrm{Mo}(1)-\mathrm{O}(2)$ & $1.9342(13)$ \\
\hline $\mathrm{Fe}(1)-\mathrm{C}(6)$ & $2.0530(17)$ \\
\hline $\mathrm{Fe}(1)-\mathrm{C}(6) \# 1$ & 2.0531(17) \\
\hline $\mathrm{Fe}(1)-\mathrm{C}(5)$ & 2.0561(19) \\
\hline $\mathrm{Fe}(1)-\mathrm{C}(5) \# 1$ & 2.0562(19) \\
\hline $\mathrm{Fe}(1)-\mathrm{C}(4)$ & 2.0566(19) \\
\hline $\mathrm{Fe}(1)-\mathrm{C}(4) \# 1$ & 2.0566(19) \\
\hline $\mathrm{Fe}(1)-\mathrm{C}(3) \# 1$ & 2.0583(18) \\
\hline $\mathrm{Fe}(1)-\mathrm{C}(3)$ & $2.0583(18)$ \\
\hline $\mathrm{Fe}(1)-\mathrm{C}(2)$ & $2.0757(17)$ \\
\hline $\mathrm{Fe}(1)-\mathrm{C}(2) \# 1$ & $2.0757(17)$ \\
\hline $\mathrm{N}(1)-\mathrm{C}(7)$ & $1.395(2)$ \\
\hline $\mathrm{O}(1)-\mathrm{C}(23)$ & $1.390(2)$ \\
\hline $\mathrm{O}(2)-\mathrm{C}(19)$ & $1.386(2)$ \\
\hline $\mathrm{C}(1)-\mathrm{C}(2)$ & $1.452(3)$ \\
\hline $\mathrm{F}(7)-\mathrm{C}(25)$ & $1.340(3)$ \\
\hline$F(5)-C(22)$ & $1.321(3)$ \\
\hline C(8)-C(9) & 1.394(3) \\
\hline $\mathrm{C}(8)-\mathrm{C}(7)$ & $1.414(3)$ \\
\hline C(8)-C(13) & 1.512(3) \\
\hline $\mathrm{C}(7)-\mathrm{C}(12)$ & $1.412(3)$ \\
\hline $\mathrm{C}(3)-\mathrm{C}(4) \# 1$ & 1.421(3) \\
\hline$C(3)-C(2)$ & $1.435(2)$ \\
\hline C(13)-C(14) & $1.530(3)$ \\
\hline C(13)-C(15) & $1.534(3)$ \\
\hline $\mathrm{F}(11)-\mathrm{C}(26)$ & $1.330(3)$ \\
\hline$F(12)-C(26)$ & $1.328(3)$ \\
\hline$C(6)-C(5)$ & $1.420(3)$ \\
\hline $\mathrm{C}(6)-\mathrm{C}(2) \# 1$ & $1.443(3)$ \\
\hline $\mathrm{C}(2)-\mathrm{C}(6) \# 1$ & $1.443(3)$ \\
\hline $\mathrm{C}(23)-\mathrm{C}(24)$ & $1.527(3)$ \\
\hline
\end{tabular}




\begin{tabular}{|c|c|}
\hline$C(23)-C(25)$ & $1.532(3)$ \\
\hline$C(23)-C(26)$ & $1.543(3)$ \\
\hline$C(12)-C(11)$ & 1.391(3) \\
\hline$C(12)-C(16)$ & $1.516(3)$ \\
\hline $\mathrm{F}(9)-\mathrm{C}(25)$ & $1.319(3)$ \\
\hline$C(5)-C(4)$ & $1.424(3)$ \\
\hline$C(19)-C(20)$ & $1.523(3)$ \\
\hline $\mathrm{C}(19)-\mathrm{C}(22)$ & $1.533(3)$ \\
\hline$C(19)-C(21)$ & $1.542(3)$ \\
\hline $\mathrm{C}(16)-\mathrm{C}(17)$ & $1.529(3)$ \\
\hline$C(16)-C(18)$ & $1.535(3)$ \\
\hline $\mathrm{F}(3)-\mathrm{C}(21)$ & $1.343(3)$ \\
\hline $\mathrm{F}(1)-\mathrm{C}(21)$ & $1.329(3)$ \\
\hline$C(25)-F(8)$ & $1.336(3)$ \\
\hline $\mathrm{C}(4)-\mathrm{C}(3) \# 1$ & $1.421(3)$ \\
\hline $\mathrm{F}(10)-\mathrm{C}(26)$ & $1.330(3)$ \\
\hline $\mathrm{F}(2)-\mathrm{C}(21)$ & $1.316(4)$ \\
\hline $\mathrm{F}(4)-\mathrm{C}(22)$ & $1.339(3)$ \\
\hline $\mathrm{F}(6)-\mathrm{C}(22)$ & $1.329(3)$ \\
\hline C(9)-C(10) & $1.382(3)$ \\
\hline $\mathrm{C}(11)-\mathrm{C}(10)$ & $1.382(4)$ \\
\hline $\mathrm{C}(28)-\mathrm{C}(27)$ & $1.372(5)$ \\
\hline $\mathrm{C}(28)-\mathrm{C}(29)$ & $1.378(5)$ \\
\hline C(29)-C(27)\#2 & $1.365(5)$ \\
\hline C(27)-C(29)\#2 & $1.365(5)$ \\
\hline $\mathrm{N}(1)-\mathrm{Mo}(1)-\mathrm{C}(1)$ & $98.79(7)$ \\
\hline $\mathrm{N}(1)-\mathrm{Mo}(1)-\mathrm{O}(1)$ & 110.93(6) \\
\hline $\mathrm{C}(1)-\mathrm{Mo}(1)-\mathrm{O}(1)$ & $105.59(6)$ \\
\hline $\mathrm{N}(1)-\mathrm{Mo}(1)-\mathrm{O}(2)$ & 119.64(6) \\
\hline $\mathrm{C}(1)-\mathrm{Mo}(1)-\mathrm{O}(2)$ & 107.90(7) \\
\hline $\mathrm{O}(1)-\mathrm{Mo}(1)-\mathrm{O}(2)$ & 112.16(6) \\
\hline $\mathrm{C}(6)-\mathrm{Fe}(1)-\mathrm{C}(6) \# 1$ & 180.0 \\
\hline $\mathrm{C}(6)-\mathrm{Fe}(1)-\mathrm{C}(5)$ & $40.43(8)$ \\
\hline C(6)\#1-Fe(1)-C(5) & 139.57(8) \\
\hline $\mathrm{C}(6)-\mathrm{Fe}(1)-\mathrm{C}(5) \# 1$ & $139.57(8)$ \\
\hline
\end{tabular}




\begin{tabular}{|c|c|}
\hline C(6)\#1-Fe(1)-C(5)\#1 & $40.43(8)$ \\
\hline $\mathrm{C}(5)-\mathrm{Fe}(1)-\mathrm{C}(5) \# 1$ & 180.0 \\
\hline $\mathrm{C}(6)-\mathrm{Fe}(1)-\mathrm{C}(4)$ & 68.01(8) \\
\hline $\mathrm{C}(6) \# 1-\mathrm{Fe}(1)-\mathrm{C}(4)$ & 111.99(8) \\
\hline $\mathrm{C}(5)-\mathrm{Fe}(1)-\mathrm{C}(4)$ & $40.50(8)$ \\
\hline $\mathrm{C}(5) \# 1-\mathrm{Fe}(1)-\mathrm{C}(4)$ & $139.50(8)$ \\
\hline $\mathrm{C}(6)-\mathrm{Fe}(1)-\mathrm{C}(4) \# 1$ & 111.99(8) \\
\hline C(6)\#1-Fe(1)-C(4)\#1 & 68.01(8) \\
\hline $\mathrm{C}(5)-\mathrm{Fe}(1)-\mathrm{C}(4) \# 1$ & $139.50(8)$ \\
\hline $\mathrm{C}(5) \# 1-\mathrm{Fe}(1)-\mathrm{C}(4) \# 1$ & $40.50(8)$ \\
\hline $\mathrm{C}(4)-\mathrm{Fe}(1)-\mathrm{C}(4) \# 1$ & 180.0 \\
\hline $\mathrm{C}(6)-\mathrm{Fe}(1)-\mathrm{C}(3) \# 1$ & $68.42(7)$ \\
\hline C(6)\#1-Fe(1)-C(3)\#1 & $111.58(7)$ \\
\hline $\mathrm{C}(5)-\mathrm{Fe}(1)-\mathrm{C}(3) \# 1$ & $68.29(8)$ \\
\hline C(5)\#1-Fe(1)-C(3)\#1 & $111.71(8)$ \\
\hline $\mathrm{C}(4)-\mathrm{Fe}(1)-\mathrm{C}(3) \# 1$ & $40.40(8)$ \\
\hline $\mathrm{C}(4) \# 1-\mathrm{Fe}(1)-\mathrm{C}(3) \# 1$ & 139.61(8) \\
\hline $\mathrm{C}(6)-\mathrm{Fe}(1)-\mathrm{C}(3)$ & $111.58(7)$ \\
\hline C(6)\#1-Fe(1)-C(3) & $68.42(7)$ \\
\hline $\mathrm{C}(5)-\mathrm{Fe}(1)-\mathrm{C}(3)$ & $111.71(8)$ \\
\hline C(5)\#1-Fe(1)-C(3) & $68.29(8)$ \\
\hline $\mathrm{C}(4)-\mathrm{Fe}(1)-\mathrm{C}(3)$ & 139.60(8) \\
\hline $\mathrm{C}(4) \# 1-\mathrm{Fe}(1)-\mathrm{C}(3)$ & 40.39(8) \\
\hline C(3)\#1-Fe(1)-C(3) & $180.00(12)$ \\
\hline $\mathrm{C}(6)-\mathrm{Fe}(1)-\mathrm{C}(2)$ & 139.10(7) \\
\hline $\mathrm{C}(6) \# 1-\mathrm{Fe}(1)-\mathrm{C}(2)$ & $40.90(7)$ \\
\hline $\mathrm{C}(5)-\mathrm{Fe}(1)-\mathrm{C}(2)$ & $111.60(7)$ \\
\hline C(5)\#1-Fe(1)-C(2) & $68.40(7)$ \\
\hline $\mathrm{C}(4)-\mathrm{Fe}(1)-\mathrm{C}(2)$ & $111.92(7)$ \\
\hline $\mathrm{C}(4) \# 1-\mathrm{Fe}(1)-\mathrm{C}(2)$ & $68.08(7)$ \\
\hline C(3)\#1-Fe(1)-C(2) & 139.39(7) \\
\hline $\mathrm{C}(3)-\mathrm{Fe}(1)-\mathrm{C}(2)$ & $40.61(7)$ \\
\hline $\mathrm{C}(6)-\mathrm{Fe}(1)-\mathrm{C}(2) \# 1$ & 40.90(7) \\
\hline $\mathrm{C}(6) \# 1-\mathrm{Fe}(1)-\mathrm{C}(2) \# 1$ & 139.10(7) \\
\hline $\mathrm{C}(5)-\mathrm{Fe}(1)-\mathrm{C}(2) \# 1$ & $68.40(7)$ \\
\hline $\mathrm{C}(5) \# 1-\mathrm{Fe}(1)-\mathrm{C}(2) \# 1$ & $111.60(7)$ \\
\hline
\end{tabular}




\begin{tabular}{|c|c|}
\hline $\mathrm{C}(4)-\mathrm{Fe}(1)-\mathrm{C}(2) \# 1$ & $68.08(7)$ \\
\hline $\mathrm{C}(4) \# 1-\mathrm{Fe}(1)-\mathrm{C}(2) \# 1$ & $111.92(7)$ \\
\hline $\mathrm{C}(3) \# 1-\mathrm{Fe}(1)-\mathrm{C}(2) \# 1$ & $40.61(7)$ \\
\hline $\mathrm{C}(3)-\mathrm{Fe}(1)-\mathrm{C}(2) \# 1$ & 139.39(7) \\
\hline $\mathrm{C}(2)-\mathrm{Fe}(1)-\mathrm{C}(2) \# 1$ & 180.0 \\
\hline $\mathrm{C}(7)-\mathrm{N}(1)-\mathrm{Mo}(1)$ & 174.37(13) \\
\hline C(23)-O(1)-Mo(1) & $142.03(12)$ \\
\hline C(19)-O(2)-Mo(1) & $148.02(13)$ \\
\hline $\mathrm{C}(2)-\mathrm{C}(1)-\mathrm{Mo}(1)$ & 139.06(13) \\
\hline C(9)-C(8)-C(7) & 116.95(19) \\
\hline C(9)-C(8)-C(13) & $122.14(18)$ \\
\hline C(7)-C(8)-C(13) & $120.87(16)$ \\
\hline $\mathrm{N}(1)-\mathrm{C}(7)-\mathrm{C}(12)$ & $118.63(16)$ \\
\hline N(1)-C(7)-C(8) & $118.55(16)$ \\
\hline C(12)-C(7)-C(8) & $122.83(17)$ \\
\hline C(4)\#1-C(3)-C(2) & $108.22(17)$ \\
\hline C(4)\#1-C(3)-Fe(1) & $69.74(11)$ \\
\hline $\mathrm{C}(2)-\mathrm{C}(3)-\mathrm{Fe}(1)$ & $70.35(10)$ \\
\hline C(8)-C(13)-C(14) & $113.3(2)$ \\
\hline$C(8)-C(13)-C(15)$ & 109.87(18) \\
\hline C(14)-C(13)-C(15) & $111.0(2)$ \\
\hline$C(5)-C(6)-C(2) \# 1$ & $108.45(16)$ \\
\hline $\mathrm{C}(5)-\mathrm{C}(6)-\mathrm{Fe}(1)$ & $69.90(11)$ \\
\hline C(2)\#1-C(6)-Fe(1) & $70.40(10)$ \\
\hline$C(3)-C(2)-C(6) \# 1$ & $106.90(16)$ \\
\hline$C(3)-C(2)-C(1)$ & 128.69(17) \\
\hline $\mathrm{C}(6) \# 1-\mathrm{C}(2)-\mathrm{C}(1)$ & 124.39(16) \\
\hline $\mathrm{C}(3)-\mathrm{C}(2)-\mathrm{Fe}(1)$ & $69.04(10)$ \\
\hline C(6)\#1-C(2)-Fe(1) & $68.71(10)$ \\
\hline $\mathrm{C}(1)-\mathrm{C}(2)-\mathrm{Fe}(1)$ & $128.42(13)$ \\
\hline $\mathrm{O}(1)-\mathrm{C}(23)-\mathrm{C}(24)$ & 113.91(16) \\
\hline $\mathrm{O}(1)-\mathrm{C}(23)-\mathrm{C}(25)$ & $107.56(16)$ \\
\hline C(24)-C(23)-C(25) & 109.82(19) \\
\hline $\mathrm{O}(1)-\mathrm{C}(23)-\mathrm{C}(26)$ & 105.81(17) \\
\hline C(24)-C(23)-C(26) & 109.07(18) \\
\hline C(25)-C(23)-C(26) & $110.60(18)$ \\
\hline
\end{tabular}




\begin{tabular}{|c|c|}
\hline $\mathrm{C}(11)-\mathrm{C}(12)-\mathrm{C}(7)$ & 117.03(19) \\
\hline$C(11)-C(12)-C(16)$ & 121.24(19) \\
\hline$C(7)-C(12)-C(16)$ & $121.70(17)$ \\
\hline $\mathrm{C}(6)-\mathrm{C}(5)-\mathrm{C}(4)$ & 107.86(17) \\
\hline $\mathrm{C}(6)-\mathrm{C}(5)-\mathrm{Fe}(1)$ & $69.67(11)$ \\
\hline $\mathrm{C}(4)-\mathrm{C}(5)-\mathrm{Fe}(1)$ & 69.77(11) \\
\hline $\mathrm{O}(2)-\mathrm{C}(19)-\mathrm{C}(20)$ & 111.65(18) \\
\hline $\mathrm{O}(2)-\mathrm{C}(19)-\mathrm{C}(22)$ & 109.26(17) \\
\hline$C(20)-C(19)-C(22)$ & $110.3(2)$ \\
\hline $\mathrm{O}(2)-\mathrm{C}(19)-\mathrm{C}(21)$ & 106.19(17) \\
\hline$C(20)-C(19)-C(21)$ & $110.0(2)$ \\
\hline$C(22)-C(19)-C(21)$ & 109.31(18) \\
\hline$C(12)-C(16)-C(17)$ & $110.1(2)$ \\
\hline$C(12)-C(16)-C(18)$ & 113.66(19) \\
\hline$C(17)-C(16)-C(18)$ & 109.72(19) \\
\hline $\mathrm{F}(9)-\mathrm{C}(25)-\mathrm{F}(8)$ & 108.1(2) \\
\hline $\mathrm{F}(9)-\mathrm{C}(25)-\mathrm{F}(7)$ & $106.7(2)$ \\
\hline $\mathrm{F}(8)-\mathrm{C}(25)-\mathrm{F}(7)$ & 106.6(2) \\
\hline F(9)-C(25)-C(23) & $113.3(2)$ \\
\hline $\mathrm{F}(8)-\mathrm{C}(25)-\mathrm{C}(23)$ & $112.0(2)$ \\
\hline F(7)-C(25)-C(23) & 109.68(19) \\
\hline C(3)\#1-C(4)-C(5) & 108.56(17) \\
\hline C(3)\#1-C(4)-Fe(1) & 69.87(11) \\
\hline $\mathrm{C}(5)-\mathrm{C}(4)-\mathrm{Fe}(1)$ & 69.73(11) \\
\hline C(10)-C(9)-C(8) & 121.1(2) \\
\hline $\mathrm{C}(10)-\mathrm{C}(11)-\mathrm{C}(12)$ & $121.2(2)$ \\
\hline $\mathrm{C}(11)-\mathrm{C}(10)-\mathrm{C}(9)$ & 120.87(19) \\
\hline$F(12)-C(26)-F(10)$ & $107.2(2)$ \\
\hline $\mathrm{F}(12)-\mathrm{C}(26)-\mathrm{F}(11)$ & 106.1(2) \\
\hline$F(10)-C(26)-F(11)$ & $107.1(2)$ \\
\hline $\mathrm{F}(12)-\mathrm{C}(26)-\mathrm{C}(23)$ & 113.08(19) \\
\hline $\mathrm{F}(10)-\mathrm{C}(26)-\mathrm{C}(23)$ & $112.6(2)$ \\
\hline $\mathrm{F}(11)-\mathrm{C}(26)-\mathrm{C}(23)$ & 110.31(18) \\
\hline$F(5)-C(22)-F(6)$ & $106.2(2)$ \\
\hline$F(5)-C(22)-F(4)$ & $106.5(2)$ \\
\hline $\mathrm{F}(6)-\mathrm{C}(22)-\mathrm{F}(4)$ & $107.6(2)$ \\
\hline
\end{tabular}




$\begin{array}{ll}\mathrm{F}(5)-\mathrm{C}(22)-\mathrm{C}(19) & 110.8(2) \\ \mathrm{F}(6)-\mathrm{C}(22)-\mathrm{C}(19) & 112.8(2) \\ \mathrm{F}(4)-\mathrm{C}(22)-\mathrm{C}(19) & 112.5(2) \\ \mathrm{F}(2)-\mathrm{C}(21)-\mathrm{F}(1) & 106.9(2) \\ \mathrm{F}(2)-\mathrm{C}(21)-\mathrm{F}(3) & 108.5(2) \\ \mathrm{F}(1)-\mathrm{C}(21)-\mathrm{F}(3) & 106.7(2) \\ \mathrm{F}(2)-\mathrm{C}(21)-\mathrm{C}(19) & 113.2(2) \\ \mathrm{F}(1)-\mathrm{C}(21)-\mathrm{C}(19) & 110.0(2) \\ \mathrm{F}(3)-\mathrm{C}(21)-\mathrm{C}(19) & 111.3(2) \\ \mathrm{C}(27)-\mathrm{C}(28)-\mathrm{C}(29) & 120.8(3) \\ \mathrm{C}(27) \# 2-\mathrm{C}(29)-\mathrm{C}(28) & 119.0(3) \\ \mathrm{C}(29) \# 2-\mathrm{C}(27)-\mathrm{C}(28) & 120.2(3)\end{array}$

Symmetry transformations used to generate equivalent atoms:

$\# 1-\mathrm{x}+1,-\mathrm{y},-\mathrm{z} \quad \# 2-\mathrm{x}+1,-\mathrm{y}+1,-\mathrm{z}+1$ 
Table 8. Anisotropic displacement parameters $\left(\AA^{2} \times 10^{3}\right)$ for 3 . The anisotropic displacement factor exponent takes the form: $-2 \pi^{2}\left[\mathbf{h}^{2} \mathbf{a}^{* 2} \mathbf{U}^{11}+\ldots+2 \mathbf{h} \mathbf{k} \mathbf{a}^{*} \mathbf{b}^{*} \mathbf{U}^{12}\right]$

\begin{tabular}{|c|c|c|c|c|c|c|}
\hline & $\mathrm{U}^{11}$ & $\mathrm{U}^{22}$ & $\mathrm{U}^{33}$ & $\mathrm{U}^{23}$ & $\mathrm{U}^{13}$ & $\mathrm{U}^{12}$ \\
\hline $\operatorname{Mo}(1)$ & $22(1)$ & $20(1)$ & $27(1)$ & 7(1) & $7(1)$ & $6(1)$ \\
\hline $\mathrm{Fe}(1)$ & $23(1)$ & $22(1)$ & 26(1) & $4(1)$ & $7(1)$ & $9(1)$ \\
\hline $\mathrm{N}(1)$ & $25(1)$ & $22(1)$ & 26(1) & $5(1)$ & $6(1)$ & 6(1) \\
\hline $\mathrm{O}(1)$ & $35(1)$ & $35(1)$ & $28(1)$ & 9(1) & $10(1)$ & $20(1)$ \\
\hline $\mathrm{O}(2)$ & $40(1)$ & $24(1)$ & 35(1) & $11(1)$ & $16(1)$ & 6(1) \\
\hline $\mathrm{C}(1)$ & $23(1)$ & $22(1)$ & 32(1) & $5(1)$ & $6(1)$ & 7(1) \\
\hline $\mathrm{F}(7)$ & $55(1)$ & $46(1)$ & 79(1) & $10(1)$ & $16(1)$ & 1(1) \\
\hline $\mathrm{F}(5)$ & $74(1)$ & $85(1)$ & $44(1)$ & $16(1)$ & $6(1)$ & $-28(1)$ \\
\hline$C(8)$ & $28(1)$ & $35(1)$ & 24(1) & $2(1)$ & $5(1)$ & $7(1)$ \\
\hline C(7) & $25(1)$ & $27(1)$ & 23(1) & $2(1)$ & $7(1)$ & 3(1) \\
\hline C(3) & $28(1)$ & $22(1)$ & 36(1) & $5(1)$ & $10(1)$ & $8(1)$ \\
\hline $\mathrm{C}(13)$ & $35(1)$ & $33(1)$ & 38(1) & $7(1)$ & $1(1)$ & $13(1)$ \\
\hline $\mathrm{F}(11)$ & $72(1)$ & $34(1)$ & $109(1)$ & $18(1)$ & $36(1)$ & $16(1)$ \\
\hline $\mathrm{F}(12)$ & $92(1)$ & $58(1)$ & 64(1) & $10(1)$ & $35(1)$ & $46(1)$ \\
\hline $\mathrm{C}(6)$ & $30(1)$ & $30(1)$ & 30(1) & $7(1)$ & $5(1)$ & $14(1)$ \\
\hline$C(2)$ & $26(1)$ & $22(1)$ & 32(1) & $6(1)$ & $8(1)$ & $10(1)$ \\
\hline$C(23)$ & $32(1)$ & $37(1)$ & $34(1)$ & $14(1)$ & $10(1)$ & $17(1)$ \\
\hline $\mathrm{C}(12)$ & $34(1)$ & $30(1)$ & 28(1) & $5(1)$ & $10(1)$ & 1(1) \\
\hline $\mathrm{F}(9)$ & $60(1)$ & $87(1)$ & 96(1) & $28(1)$ & $52(1)$ & $21(1)$ \\
\hline$C(5)$ & $43(1)$ & $33(1)$ & 30(1) & $11(1)$ & $14(1)$ & $19(1)$ \\
\hline$C(19)$ & $36(1)$ & $24(1)$ & 39(1) & $10(1)$ & $15(1)$ & $6(1)$ \\
\hline$C(16)$ & $43(1)$ & $27(1)$ & $42(1)$ & 13(1) & 11(1) & $4(1)$ \\
\hline $\mathrm{F}(3)$ & $92(1)$ & $70(1)$ & 71(1) & $46(1)$ & $10(1)$ & $-18(1)$ \\
\hline $\mathrm{F}(1)$ & $72(1)$ & $83(1)$ & 53(1) & $34(1)$ & $-4(1)$ & $-11(1)$ \\
\hline$C(25)$ & $35(1)$ & $59(2)$ & $59(2)$ & $24(1)$ & $13(1)$ & $17(1)$ \\
\hline$C(4)$ & $34(1)$ & $29(1)$ & $42(1)$ & 13(1) & $17(1)$ & $11(1)$ \\
\hline $\mathrm{F}(10)$ & 101(1) & $77(1)$ & 85(1) & $33(1)$ & $12(1)$ & $67(1)$ \\
\hline $\mathrm{F}(2)$ & $96(1)$ & $100(2)$ & 71(1) & $15(1)$ & $57(1)$ & $28(1)$ \\
\hline $\mathrm{F}(4)$ & $69(1)$ & 57(1) & 69(1) & $18(1)$ & $12(1)$ & $-27(1)$ \\
\hline $\mathrm{C}(15)$ & $43(1)$ & $50(1)$ & $49(1)$ & $22(1)$ & $9(1)$ & $8(1)$ \\
\hline $\mathrm{F}(6)$ & $47(1)$ & $125(2)$ & $104(2)$ & $28(1)$ & $13(1)$ & $38(1)$ \\
\hline
\end{tabular}




$\begin{array}{lcccccc}\mathrm{C}(9) & 28(1) & 56(1) & 35(1) & 8(1) & 4(1) & 11(1) \\ \mathrm{C}(11) & 38(1) & 40(1) & 43(1) & 12(1) & 10(1) & -8(1) \\ \mathrm{C}(10) & 26(1) & 59(2) & 42(1) & 8(1) & 5(1) & -5(1) \\ \mathrm{F}(8) & 37(1) & 120(2) & 117(2) & 63(1) & -6(1) & 20(1) \\ \mathrm{C}(24) & 51(1) & 56(1) & 35(1) & 19(1) & 15(1) & 25(1) \\ \mathrm{C}(26) & 54(1) & 47(1) & 56(1) & 20(1) & 19(1) & 32(1) \\ \mathrm{C}(22) & 45(1) & 49(1) & 47(1) & 13(1) & 14(1) & -7(1) \\ \mathrm{C}(21) & 55(2) & 51(1) & 48(1) & 26(1) & 12(1) & -3(1) \\ \mathrm{C}(17) & 79(2) & 56(2) & 41(1) & 18(1) & 9(1) & 24(1) \\ \mathrm{C}(14) & 60(2) & 56(2) & 84(2) & 16(2) & 10(2) & 37(1) \\ \mathrm{C}(20) & 66(2) & 32(1) & 123(3) & 17(2) & 46(2) & 19(1) \\ \mathrm{C}(28) & 109(3) & 54(2) & 63(2) & 9(2) & 50(2) & 12(2) \\ \mathrm{C}(29) & 94(2) & 72(2) & 45(2) & 4(1) & 33(2) & 24(2) \\ \mathrm{C}(18) & 74(2) & 32(1) & 58(2) & 15(1) & 18(1) & 12(1) \\ \mathrm{C}(27) & 111(3) & 76(2) & 53(2) & 24(2) & 40(2) & 34(2) \\ \end{array}$


Table 9. Crystal data and structure refinement for 4.

Empirical formula

Formula weight

Temperature

Wavelength

Crystal system

Space group

Unit cell dimensions

Volume

$\mathrm{Z}$

Density (calculated)

Absorption coefficient

$\mathrm{F}(000)$

Crystal size

Theta range for data collection

Index ranges

Reflections collected

Independent reflections

Completeness to theta $=25.03^{\circ}$

Max. and min. transmission

Refinement method

Data / restraints / parameters

Goodness-of-fit on $\mathrm{F}^{2}$

Final R indices [I $>2$ sigma(I)]

$\mathrm{R}$ indices (all data)

Largest diff. peak and hole
C59 H88 Fe Mo2 N2 O4

1137.04

373(2) K

$0.71073 \AA$

Monoclinic

$\mathrm{C} 2 / \mathrm{c}$

$$
\begin{array}{ll}
\mathrm{a}=26.209(5) \AA & \alpha=90^{\circ} . \\
\mathrm{b}=10.958(2) \AA & \beta=101.62(3)^{\circ} . \\
\mathrm{c}=20.732(4) \AA & \gamma=90^{\circ} .
\end{array}
$$

\section{2(2) $\AA^{3}$}

\section{4}

$1.295 \mathrm{Mg} / \mathrm{m}^{3}$

$0.713 \mathrm{~mm}^{-1}$

2392

$0.08 \times 0.08 \times 0.05 \mathrm{~mm}^{3}$

1.59 to $25.03^{\circ}$.

$-31<=\mathrm{h}<=31,-12<=\mathrm{k}<=13,-24<=\mathrm{l}<=24$

32410

$5110[\mathrm{R}$ (int) $=0.0891]$

$99.4 \%$

0.9652 and 0.9452

Full-matrix least-squares on $\mathrm{F}^{2}$

5110 / 0 / 310

1.320

$\mathrm{R} 1=0.0826, \mathrm{wR} 2=0.1624$

$\mathrm{R} 1=0.0923, \mathrm{wR} 2=0.1659$

1.296 and -1.931 e. $\AA^{-3}$ 
Table 10. Atomic coordinates $\left(x 1^{4}\right)$ and equivalent isotropic displacement parameters $\left(\AA^{2} \times 10^{3}\right)$ for 4 . $U(e q)$ is defined as one third of the trace of the orthogonalized $U^{i j}$ tensor.

\begin{tabular}{|c|c|c|c|c|}
\hline & $\mathrm{x}$ & $\mathrm{y}$ & $\mathrm{z}$ & $\mathrm{U}(\mathrm{eq})$ \\
\hline $\mathrm{Mo}(1)$ & 1359(1) & 2796(1) & $4875(1)$ & $9(1)$ \\
\hline $\mathrm{Fe}(2)$ & 0 & 0 & 5000 & 11(1) \\
\hline $\mathrm{O}(1)$ & $1372(2)$ & $4430(5)$ & $4588(2)$ & $15(1)$ \\
\hline $\mathrm{O}(2)$ & $1825(2)$ & $1838(4)$ & $4499(2)$ & $14(1)$ \\
\hline $\mathrm{N}(1)$ & $1412(2)$ & 2655(5) & 5719(3) & $11(1)$ \\
\hline $\mathrm{C}(1)$ & 668(3) & $2185(7)$ & $4587(3)$ & $14(1)$ \\
\hline $\mathrm{C}(7)$ & 1024(3) & $5381(7)$ & $4300(4)$ & $18(2)$ \\
\hline$C(24)$ & 1848(3) & $341(7)$ & $6175(4)$ & $17(2)$ \\
\hline$C(15)$ & $1583(2)$ & $2510(6)$ & 6402(3) & $8(1)$ \\
\hline $\mathrm{C}(6)$ & $-256(3)$ & $-1501(6)$ & $4439(3)$ & $12(1)$ \\
\hline $\mathrm{C}(11)$ & 1836(3) & 1023(7) & 3968(3) & $17(2)$ \\
\hline$C(18)$ & 1961(3) & $2240(8)$ & 7738(3) & $22(2)$ \\
\hline$C(13)$ & $2405(3)$ & $610(8)$ & $4035(4)$ & $21(2)$ \\
\hline$C(16)$ & 1551(3) & $3502(6)$ & 6822(3) & $12(1)$ \\
\hline$C(21)$ & 1298(3) & $4681(7)$ & $6546(3)$ & $17(2)$ \\
\hline $\mathrm{C}(2)$ & 244(3) & 1788(6) & $4878(3)$ & $14(2)$ \\
\hline C(3) & $-291(3)$ & $1581(7)$ & $4540(4)$ & $16(2)$ \\
\hline$C(20)$ & 1796(3) & $1384(6)$ & 6641(3) & 13(1) \\
\hline$C(5)$ & 249(3) & $-1177(7)$ & $4361(4)$ & $17(2)$ \\
\hline $\mathrm{C}(8)$ & 1355(3) & $6510(7)$ & $4287(4)$ & $24(2)$ \\
\hline C(19) & 1983(3) & 1266(7) & 7316(3) & $16(2)$ \\
\hline$C(17)$ & 1745(3) & $3340(7)$ & 7494(3) & $17(2)$ \\
\hline$C(22)$ & 704(3) & $4590(7)$ & $6479(4)$ & $25(2)$ \\
\hline $\mathrm{C}(9)$ & 613(3) & 5582(8) & $4716(4)$ & $22(2)$ \\
\hline$C(10)$ & $769(3)$ & $5000(8)$ & $3602(4)$ & $29(2)$ \\
\hline$C(25)$ & 1750(3) & $-919(7)$ & $6440(4)$ & $23(2)$ \\
\hline$C(23)$ & 1498(3) & $5820(7)$ & $6943(4)$ & $24(2)$ \\
\hline $\mathrm{C}(12)$ & 1487(3) & $-59(8)$ & $4015(4)$ & $27(2)$ \\
\hline$C(26)$ & 2382(3) & 388(8) & $5982(4)$ & $23(2)$ \\
\hline$C(14)$ & 1657(3) & $1717(8)$ & $3326(4)$ & $27(2)$ \\
\hline
\end{tabular}




\begin{tabular}{lccll}
$\mathrm{C}(4)$ & $-587(3)$ & $1218(7)$ & $5012(4)$ & $17(2)$ \\
$\mathrm{C}(1 S)$ & 10000 & $-2000(13)$ & 2500 & $36(3)$ \\
$\mathrm{C}(5 S)$ & 10000 & $546(13)$ & 2500 & $37(3)$ \\
$\mathrm{C}(2 S)$ & $9574(3)$ & $-1383(9)$ & $2594(4)$ & $33(2)$ \\
$\mathrm{C}(4 S)$ & $9569(4)$ & $-130(9)$ & $2604(4)$ & $35(2)$ \\
$\mathrm{C}(3 S)$ & 10000 & $1916(13)$ & 2500 & $59(5)$ \\
\hline
\end{tabular}


Table 11. Bond lengths $[\AA ̊]$ and angles $\left[^{\circ}\right]$ for 4.

\begin{tabular}{|c|c|}
\hline $\mathrm{Mo}(1)-\mathrm{N}(1)$ & $1.734(5)$ \\
\hline $\mathrm{Mo}(1)-\mathrm{O}(1)$ & $1.889(5)$ \\
\hline $\mathrm{Mo}(1)-\mathrm{O}(2)$ & $1.892(5)$ \\
\hline $\operatorname{Mo}(1)-C(1)$ & $1.912(7)$ \\
\hline $\mathrm{Fe}(2)-\mathrm{C}(4) \# 1$ & $2.041(7)$ \\
\hline $\mathrm{Fe}(2)-\mathrm{C}(4)$ & $2.041(7)$ \\
\hline $\mathrm{Fe}(2)-\mathrm{C}(5)$ & $2.048(7)$ \\
\hline $\mathrm{Fe}(2)-\mathrm{C}(5) \# 1$ & $2.048(7)$ \\
\hline $\mathrm{Fe}(2)-\mathrm{C}(3)$ & $2.049(7)$ \\
\hline $\mathrm{Fe}(2)-\mathrm{C}(3) \# 1$ & $2.049(7)$ \\
\hline $\mathrm{Fe}(2)-\mathrm{C}(6)$ & $2.048(7)$ \\
\hline $\mathrm{Fe}(2)-\mathrm{C}(6) \# 1$ & $2.048(7)$ \\
\hline $\mathrm{Fe}(2)-\mathrm{C}(2)$ & $2.092(7)$ \\
\hline $\mathrm{Fe}(2)-\mathrm{C}(2) \# 1$ & $2.092(7)$ \\
\hline $\mathrm{O}(1)-\mathrm{C}(7)$ & $1.434(8)$ \\
\hline $\mathrm{O}(2)-\mathrm{C}(11)$ & $1.422(8)$ \\
\hline $\mathrm{N}(1)-\mathrm{C}(15)$ & $1.407(8)$ \\
\hline$C(1)-C(2)$ & $1.435(10)$ \\
\hline $\mathrm{C}(7)-\mathrm{C}(8)$ & $1.514(10)$ \\
\hline $\mathrm{C}(7)-\mathrm{C}(9)$ & $1.524(10)$ \\
\hline $\mathrm{C}(7)-\mathrm{C}(10)$ & $1.525(11)$ \\
\hline $\mathrm{C}(24)-\mathrm{C}(20)$ & $1.520(10)$ \\
\hline $\mathrm{C}(24)-\mathrm{C}(25)$ & $1.527(10)$ \\
\hline $\mathrm{C}(24)-\mathrm{C}(26)$ & $1.532(10)$ \\
\hline $\mathrm{C}(15)-\mathrm{C}(20)$ & $1.402(9)$ \\
\hline$C(15)-C(16)$ & $1.405(9)$ \\
\hline $\mathrm{C}(6)-\mathrm{C}(5)$ & $1.412(10)$ \\
\hline $\mathrm{C}(6)-\mathrm{C}(2) \# 1$ & $1.444(9)$ \\
\hline $\mathrm{C}(11)-\mathrm{C}(12)$ & $1.511(11)$ \\
\hline $\mathrm{C}(11)-\mathrm{C}(14)$ & $1.524(10)$ \\
\hline $\mathrm{C}(11)-\mathrm{C}(13)$ & $1.539(10)$ \\
\hline $\mathrm{C}(18)-\mathrm{C}(17)$ & $1.383(11)$ \\
\hline C(18)-C(19) & $1.388(11)$ \\
\hline $\mathrm{C}(16)-\mathrm{C}(17)$ & $1.395(10)$ \\
\hline
\end{tabular}




\begin{tabular}{|c|c|}
\hline$C(16)-C(21)$ & $1.510(10)$ \\
\hline$C(21)-C(23)$ & $1.529(10)$ \\
\hline $\mathrm{C}(21)-\mathrm{C}(22)$ & $1.540(10)$ \\
\hline $\mathrm{C}(2)-\mathrm{C}(6) \# 1$ & $1.444(9)$ \\
\hline $\mathrm{C}(2)-\mathrm{C}(3)$ & $1.452(9)$ \\
\hline $\mathrm{C}(3)-\mathrm{C}(4)$ & $1.424(10)$ \\
\hline$C(20)-C(19)$ & $1.394(10)$ \\
\hline $\mathrm{C}(5)-\mathrm{C}(4) \# 1$ & $1.417(10)$ \\
\hline$C(4)-C(5) \# 1$ & $1.417(10)$ \\
\hline $\mathrm{C}(1 \mathrm{~S})-\mathrm{C}(2 \mathrm{~S}) \# 2$ & $1.354(11)$ \\
\hline $\mathrm{C}(1 \mathrm{~S})-\mathrm{C}(2 \mathrm{~S})$ & $1.354(11)$ \\
\hline $\mathrm{C}(5 \mathrm{~S})-\mathrm{C}(4 \mathrm{~S})$ & $1.402(12)$ \\
\hline $\mathrm{C}(5 \mathrm{~S})-\mathrm{C}(4 \mathrm{~S}) \# 2$ & $1.402(12)$ \\
\hline $\mathrm{C}(5 \mathrm{~S})-\mathrm{C}(3 \mathrm{~S})$ & $1.502(19)$ \\
\hline $\mathrm{C}(2 \mathrm{~S})-\mathrm{C}(4 \mathrm{~S})$ & $1.374(13)$ \\
\hline $\mathrm{N}(1)-\mathrm{Mo}(1)-\mathrm{O}(1)$ & $113.6(2)$ \\
\hline $\mathrm{N}(1)-\mathrm{Mo}(1)-\mathrm{O}(2)$ & $116.0(2)$ \\
\hline $\mathrm{O}(1)-\mathrm{Mo}(1)-\mathrm{O}(2)$ & $110.0(2)$ \\
\hline $\mathrm{N}(1)-\mathrm{Mo}(1)-\mathrm{C}(1)$ & $99.3(3)$ \\
\hline $\mathrm{O}(1)-\mathrm{Mo}(1)-\mathrm{C}(1)$ & $108.0(3)$ \\
\hline $\mathrm{O}(2)-\mathrm{Mo}(1)-\mathrm{C}(1)$ & 109.1(3) \\
\hline $\mathrm{C}(4) \# 1-\mathrm{Fe}(2)-\mathrm{C}(4)$ & 179.999(1) \\
\hline $\mathrm{C}(4) \# 1-\mathrm{Fe}(2)-\mathrm{C}(5)$ & $40.6(3)$ \\
\hline $\mathrm{C}(4)-\mathrm{Fe}(2)-\mathrm{C}(5)$ & 139.4(3) \\
\hline $\mathrm{C}(4) \# 1-\mathrm{Fe}(2)-\mathrm{C}(5) \# 1$ & 139.4(3) \\
\hline $\mathrm{C}(4)-\mathrm{Fe}(2)-\mathrm{C}(5) \# 1$ & $40.6(3)$ \\
\hline $\mathrm{C}(5)-\mathrm{Fe}(2)-\mathrm{C}(5) \# 1$ & $180.0(3)$ \\
\hline $\mathrm{C}(4) \# 1-\mathrm{Fe}(2)-\mathrm{C}(3)$ & 139.3(3) \\
\hline $\mathrm{C}(4)-\mathrm{Fe}(2)-\mathrm{C}(3)$ & $40.7(3)$ \\
\hline $\mathrm{C}(5)-\mathrm{Fe}(2)-\mathrm{C}(3)$ & 111.6(3) \\
\hline C(5)\#1-Fe(2)-C(3) & $68.4(3)$ \\
\hline $\mathrm{C}(4) \# 1-\mathrm{Fe}(2)-\mathrm{C}(3) \# 1$ & $40.7(3)$ \\
\hline $\mathrm{C}(4)-\mathrm{Fe}(2)-\mathrm{C}(3) \# 1$ & 139.3(3) \\
\hline $\mathrm{C}(5)-\mathrm{Fe}(2)-\mathrm{C}(3) \# 1$ & $68.4(3)$ \\
\hline $\mathrm{C}(5) \# 1-\mathrm{Fe}(2)-\mathrm{C}(3) \# 1$ & 111.6(3) \\
\hline
\end{tabular}




\begin{tabular}{|c|c|}
\hline $\mathrm{C}(3)-\mathrm{Fe}(2)-\mathrm{C}(3) \# 1$ & $180.0(4)$ \\
\hline $\mathrm{C}(4) \# 1-\mathrm{Fe}(2)-\mathrm{C}(6)$ & $68.2(3)$ \\
\hline $\mathrm{C}(4)-\mathrm{Fe}(2)-\mathrm{C}(6)$ & 111.8(3) \\
\hline $\mathrm{C}(5)-\mathrm{Fe}(2)-\mathrm{C}(6)$ & $40.3(3)$ \\
\hline $\mathrm{C}(5) \# 1-\mathrm{Fe}(2)-\mathrm{C}(6)$ & 139.7(3) \\
\hline $\mathrm{C}(3)-\mathrm{Fe}(2)-\mathrm{C}(6)$ & 111.5(3) \\
\hline $\mathrm{C}(3) \# 1-\mathrm{Fe}(2)-\mathrm{C}(6)$ & $68.5(3)$ \\
\hline $\mathrm{C}(4) \# 1-\mathrm{Fe}(2)-\mathrm{C}(6) \# 1$ & 111.8(3) \\
\hline $\mathrm{C}(4)-\mathrm{Fe}(2)-\mathrm{C}(6) \# 1$ & $68.2(3)$ \\
\hline $\mathrm{C}(5)-\mathrm{Fe}(2)-\mathrm{C}(6) \# 1$ & 139.7(3) \\
\hline $\mathrm{C}(5) \# 1-\mathrm{Fe}(2)-\mathrm{C}(6) \# 1$ & $40.3(3)$ \\
\hline $\mathrm{C}(3)-\mathrm{Fe}(2)-\mathrm{C}(6) \# 1$ & $68.5(3)$ \\
\hline $\mathrm{C}(3) \# 1-\mathrm{Fe}(2)-\mathrm{C}(6) \# 1$ & 111.5(3) \\
\hline $\mathrm{C}(6)-\mathrm{Fe}(2)-\mathrm{C}(6) \# 1$ & 179.997(1) \\
\hline $\mathrm{C}(4) \# 1-\mathrm{Fe}(2)-\mathrm{C}(2)$ & 111.2(3) \\
\hline $\mathrm{C}(4)-\mathrm{Fe}(2)-\mathrm{C}(2)$ & $68.8(3)$ \\
\hline $\mathrm{C}(5)-\mathrm{Fe}(2)-\mathrm{C}(2)$ & 111.5(3) \\
\hline $\mathrm{C}(5) \# 1-\mathrm{Fe}(2)-\mathrm{C}(2)$ & $68.5(3)$ \\
\hline $\mathrm{C}(3)-\mathrm{Fe}(2)-\mathrm{C}(2)$ & $41.0(3)$ \\
\hline $\mathrm{C}(3) \# 1-\mathrm{Fe}(2)-\mathrm{C}(2)$ & $139.0(3)$ \\
\hline $\mathrm{C}(6)-\mathrm{Fe}(2)-\mathrm{C}(2)$ & 139.2(3) \\
\hline $\mathrm{C}(6) \# 1-\mathrm{Fe}(2)-\mathrm{C}(2)$ & $40.8(3)$ \\
\hline $\mathrm{C}(4) \# 1-\mathrm{Fe}(2)-\mathrm{C}(2) \# 1$ & $68.8(3)$ \\
\hline $\mathrm{C}(4)-\mathrm{Fe}(2)-\mathrm{C}(2) \# 1$ & 111.2(3) \\
\hline $\mathrm{C}(5)-\mathrm{Fe}(2)-\mathrm{C}(2) \# 1$ & $68.5(3)$ \\
\hline $\mathrm{C}(5) \# 1-\mathrm{Fe}(2)-\mathrm{C}(2) \# 1$ & $111.5(3)$ \\
\hline $\mathrm{C}(3)-\mathrm{Fe}(2)-\mathrm{C}(2) \# 1$ & $139.0(3)$ \\
\hline $\mathrm{C}(3) \# 1-\mathrm{Fe}(2)-\mathrm{C}(2) \# 1$ & $41.0(3)$ \\
\hline $\mathrm{C}(6)-\mathrm{Fe}(2)-\mathrm{C}(2) \# 1$ & $40.8(3)$ \\
\hline $\mathrm{C}(6) \# 1-\mathrm{Fe}(2)-\mathrm{C}(2) \# 1$ & 139.2(3) \\
\hline $\mathrm{C}(2)-\mathrm{Fe}(2)-\mathrm{C}(2) \# 1$ & 180.0 \\
\hline $\mathrm{C}(7)-\mathrm{O}(1)-\mathrm{Mo}(1)$ & $140.3(4)$ \\
\hline $\mathrm{C}(11)-\mathrm{O}(2)-\mathrm{Mo}(1)$ & $141.4(4)$ \\
\hline $\mathrm{C}(15)-\mathrm{N}(1)-\mathrm{Mo}(1)$ & $166.2(5)$ \\
\hline $\mathrm{C}(2)-\mathrm{C}(1)-\mathrm{Mo}(1)$ & $137.8(5)$ \\
\hline $\mathrm{O}(1)-\mathrm{C}(7)-\mathrm{C}(8)$ & $106.4(6)$ \\
\hline
\end{tabular}




$\begin{array}{lr}\mathrm{O}(1)-\mathrm{C}(7)-\mathrm{C}(9) & 109.4(6) \\ \mathrm{C}(8)-\mathrm{C}(7)-\mathrm{C}(9) & 111.3(6) \\ \mathrm{O}(1)-\mathrm{C}(7)-\mathrm{C}(10) & 108.4(6) \\ \mathrm{C}(8)-\mathrm{C}(7)-\mathrm{C}(10) & 110.5(6) \\ \mathrm{C}(9)-\mathrm{C}(7)-\mathrm{C}(10) & 110.7(6) \\ \mathrm{C}(20)-\mathrm{C}(24)-\mathrm{C}(25) & 114.1(6) \\ \mathrm{C}(20)-\mathrm{C}(24)-\mathrm{C}(26) & 110.1(6) \\ \mathrm{C}(25)-\mathrm{C}(24)-\mathrm{C}(26) & 110.6(6) \\ \mathrm{N}(1)-\mathrm{C}(15)-\mathrm{C}(20) & 118.5(6) \\ \mathrm{N}(1)-\mathrm{C}(15)-\mathrm{C}(16) & 119.3(6) \\ \mathrm{C}(20)-\mathrm{C}(15)-\mathrm{C}(16) & 122.1(6) \\ \mathrm{C}(5)-\mathrm{C}(6)-\mathrm{C}(2) \# 1 & 109.3(6) \\ \mathrm{C}(5)-\mathrm{C}(6)-\mathrm{Fe}(2) & 69.8(4) \\ \mathrm{C}(2) \# 1-\mathrm{C}(6)-\mathrm{Fe}(2) & 71.3(4) \\ \mathrm{O}(2)-\mathrm{C}(11)-\mathrm{C}(12) & 109.7(6) \\ \mathrm{O}(2)-\mathrm{C}(11)-\mathrm{C}(14) & 108.2(6) \\ \mathrm{C}(12)-\mathrm{C}(11)-\mathrm{C}(14) & 111.3(7) \\ \mathrm{O}(2)-\mathrm{C}(11)-\mathrm{C}(13) & 106.6(6) \\ \mathrm{C}(12)-\mathrm{C}(11)-\mathrm{C}(13) & 110.6(6) \\ \mathrm{C}(14)-\mathrm{C}(11)-\mathrm{C}(13) & 110.3(6) \\ \mathrm{C}(17)-\mathrm{C}(18)-\mathrm{C}(19) & 120.4(6) \\ \mathrm{C}(17)-\mathrm{C}(16)-\mathrm{C}(15) & 117.6(7) \\ \mathrm{C}(17)-\mathrm{C}(16)-\mathrm{C}(21) & 122.1(6) \\ \mathrm{C}(15)-\mathrm{C}(16)-\mathrm{C}(21) & 120.2(6) \\ \mathrm{C}(16)-\mathrm{C}(21)-\mathrm{C}(23) & 114.7(6) \\ \mathrm{C}(16)-\mathrm{C}(21)-\mathrm{C}(22) & 109.7(6) \\ \mathrm{C}(23)-\mathrm{C}(21)-\mathrm{C}(22) & 109.4(6) \\ \mathrm{C}(1)-\mathrm{C}(2)-\mathrm{C}(6) \# 1 & 127.7(6) \\ \mathrm{C}(1)-\mathrm{C}(2)-\mathrm{C}(3) & 126.7(6) \\ \mathrm{C}(6) \# 1-\mathrm{C}(2)-\mathrm{C}(3) & 105.6(6) \\ \mathrm{C}(1)-\mathrm{C}(2)-\mathrm{Fe}(2) & 128.0(5) \\ \mathrm{C}(6) \# 1-\mathrm{C}(2)-\mathrm{Fe}(2) & 67.9(4) \\ \mathrm{C}(3)-\mathrm{C}(2)-\mathrm{Fe}(2) & 6(3)-\mathrm{C}(2) \\ \end{array}$




$\begin{array}{lr}\text { C(19)-C(20)-C(15) } & 118.1(7) \\ \text { C(19)-C(20)-C(24) } & 120.7(6) \\ \text { C(15)-C(20)-C(24) } & 121.2(6) \\ \text { C(6)-C(5)-C(4)\#1 } & 108.3(6) \\ \text { C(6)-C(5)-Fe(2) } & 69.8(4) \\ \text { C(4)\#1-C(5)-Fe(2) } & 69.4(4) \\ \text { C(18)-C(19)-C(20) } & 120.7(7) \\ \text { C(18)-C(17)-C(16) } & 121.1(7) \\ \text { C(5)\#1-C(4)-C(3) } & 108.2(6) \\ \text { C(5)\#1-C(4)-Fe(2) } & 70.0(4) \\ \text { C(3)-C(4)-Fe(2) } & 70.0(4) \\ \text { C(2S)\#2-C(1S)-C(2S) } & 120.0(13) \\ \text { C(4S)-C(5S)-C(4S)\#2 } & 116.3(13) \\ \text { C(4S)-C(5S)-C(3S) } & 121.9(6) \\ \text { C(4S)\#2-C(5S)-C(3S) } & 121.9(6) \\ \text { C(1S)-C(2S)-C(4S) } & 120.7(9) \\ \text { C(2S)-C(4S)-C(5S) } & 121.1(9)\end{array}$

Symmetry transformations used to generate equivalent atoms:

$\# 1-x,-y,-z+1 \quad \# 2-x+2, y,-z+1 / 2$ 
Table 12. Anisotropic displacement parameters $\left(\AA^{2} \times 10^{3}\right)$ for 4 . The anisotropic displacement factor exponent takes the form: $-2 \pi^{2}\left[h^{2} a^{* 2} U^{11}+\ldots+2 h \mathbf{k}^{*} \mathbf{b}^{*} \mathbf{U}^{12}\right]$

\begin{tabular}{lcccccc}
\hline & $\mathrm{U} 11$ & $\mathrm{U}^{22}$ & $\mathrm{U} 33$ & $\mathrm{U}^{23}$ & $\mathrm{U}^{13}$ & $\mathrm{U} 12$ \\
\hline $\mathrm{Mo}(1)$ & $8(1)$ & $9(1)$ & $10(1)$ & $0(1)$ & $2(1)$ & $-1(1)$ \\
$\mathrm{Fe}(2)$ & $9(1)$ & $9(1)$ & $15(1)$ & $-1(1)$ & $4(1)$ & $-1(1)$ \\
$\mathrm{O}(1)$ & $5(2)$ & $19(3)$ & $21(3)$ & $2(2)$ & $4(2)$ & $0(2)$ \\
$\mathrm{O}(2)$ & $15(2)$ & $10(2)$ & $18(3)$ & $-4(2)$ & $8(2)$ & $0(2)$ \\
$\mathrm{N}(1)$ & $10(3)$ & $10(3)$ & $13(3)$ & $0(2)$ & $2(2)$ & $-4(2)$ \\
$\mathrm{C}(1)$ & $20(4)$ & $10(3)$ & $15(3)$ & $0(3)$ & $5(3)$ & $2(3)$ \\
$\mathrm{C}(7)$ & $20(4)$ & $10(4)$ & $22(4)$ & $5(3)$ & $5(3)$ & $3(3)$ \\
$\mathrm{C}(24)$ & $16(4)$ & $15(4)$ & $18(4)$ & $-1(3)$ & $-1(3)$ & $5(3)$ \\
$\mathrm{C}(15)$ & $7(3)$ & $5(4)$ & $11(3)$ & $1(2)$ & $2(2)$ & $-4(2)$ \\
$\mathrm{C}(6)$ & $9(3)$ & $14(4)$ & $12(3)$ & $-3(3)$ & $5(3)$ & $-8(3)$ \\
$\mathrm{C}(11)$ & $17(4)$ & $19(4)$ & $15(4)$ & $-3(3)$ & $6(3)$ & $-2(3)$ \\
$\mathrm{C}(18)$ & $22(4)$ & $28(4)$ & $10(3)$ & $3(3)$ & $-6(3)$ & $-6(4)$ \\
$\mathrm{C}(13)$ & $12(4)$ & $27(4)$ & $24(4)$ & $-9(3)$ & $8(3)$ & $-6(3)$ \\
$\mathrm{C}(16)$ & $7(3)$ & $14(4)$ & $15(3)$ & $1(3)$ & $5(3)$ & $-6(3)$ \\
$\mathrm{C}(21)$ & $18(4)$ & $20(4)$ & $14(4)$ & $0(3)$ & $4(3)$ & $4(3)$ \\
$\mathrm{C}(2)$ & $9(3)$ & $11(4)$ & $19(4)$ & $2(3)$ & $0(3)$ & $-3(3)$ \\
$\mathrm{C}(3)$ & $13(4)$ & $14(4)$ & $20(4)$ & $-2(3)$ & $3(3)$ & $-4(3)$ \\
$\mathrm{C}(20)$ & $9(3)$ & $10(4)$ & $19(4)$ & $4(3)$ & $3(3)$ & $-3(3)$ \\
$\mathrm{C}(5)$ & $24(4)$ & $10(4)$ & $21(4)$ & $-1(3)$ & $12(3)$ & $3(3)$ \\
$\mathrm{C}(8)$ & $22(4)$ & $18(4)$ & $34(5)$ & $2(4)$ & $13(4)$ & $3(3)$ \\
$\mathrm{C}(19)$ & $17(4)$ & $14(4)$ & $14(4)$ & $4(3)$ & $1(3)$ & $-1(3)$ \\
$\mathrm{C}(17)$ & $16(4)$ & $20(4)$ & $16(4)$ & $-6(3)$ & $5(3)$ & $-6(3)$ \\
$\mathrm{C}(22)$ & $22(4)$ & $19(4)$ & $31(4)$ & $-2(3)$ & $1(3)$ & $6(3)$ \\
$\mathrm{C}(9)$ & $18(4)$ & $25(4)$ & $24(4)$ & $4(3)$ & $8(3)$ & $3(3)$ \\
$\mathrm{C}(10)$ & $29(5)$ & $37(5)$ & $20(4)$ & $9(4)$ & $6(3)$ & $8(4)$ \\
$\mathrm{C}(25)$ & $33(5)$ & $14(4)$ & $23(4)$ & $6(3)$ & $5(3)$ & $2(3)$ \\
$\mathrm{C}(23)$ & $26(4)$ & $19(4)$ & $27(4)$ & $-1(3)$ & $4(3)$ & $-4(3)$ \\
$\mathrm{C}(12)$ & $18(4)$ & $27(5)$ & $41(5)$ & $-19(4)$ & $15(4)$ & $-4(4)$ \\
$\mathrm{C}(26)$ & $18(4)$ & $25(5)$ & $25(4)$ & $4(3)$ & $3(3)$ & $10(3)$ \\
$\mathrm{C}(14)$ & $36(5)$ & $32(5)$ & $13(4)$ & $-1(3)$ & $4(3)$ & $1(4)$ \\
$\mathrm{C}(4)$ & $10(4)$ & $24(4)$ & $19(4)$ & $0(3)$ & $7(3)$ & $1(3)$ \\
& & & & & &
\end{tabular}




$\begin{array}{lcccccc}\mathrm{C}(1 \mathrm{~S}) & 43(8) & 35(8) & 27(6) & 0 & -2(6) & 0 \\ \mathrm{C}(5 \mathrm{~S}) & 47(8) & 35(8) & 23(6) & 0 & -8(6) & 0 \\ \mathrm{C}(2 \mathrm{~S}) & 24(5) & 45(6) & 29(5) & 5(4) & 0(4) & -13(4) \\ \mathrm{C}(4 \mathrm{~S}) & 28(5) & 52(6) & 26(5) & 4(4) & 4(4) & 11(5) \\ \mathrm{C}(3 S) & 77(12) & 37(9) & 47(9) & 0 & -24(8) & 0\end{array}$


